
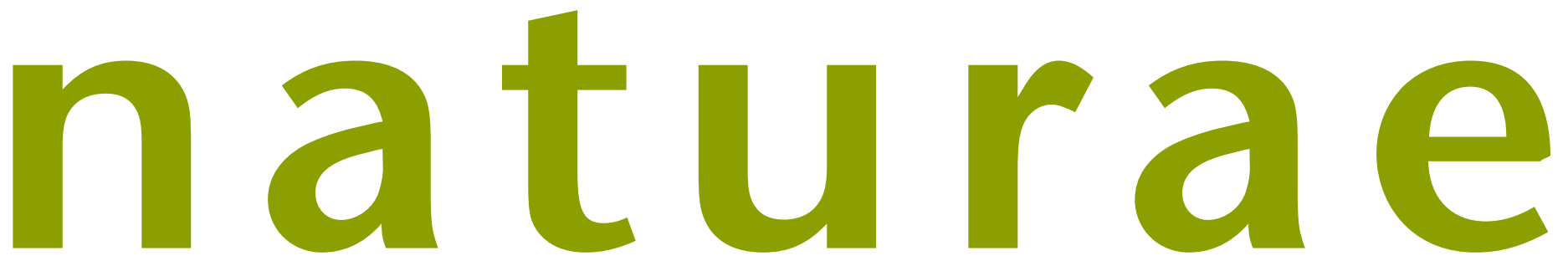

$2021 \cdot 13$

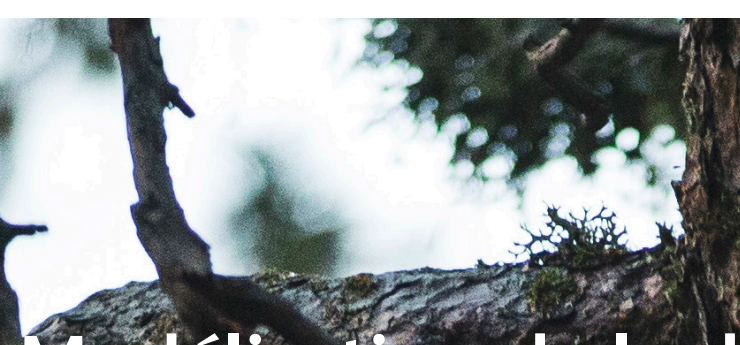

Mocélisation de la distribution des petites chouettes de montagne dans les Alpes du Nord françaises

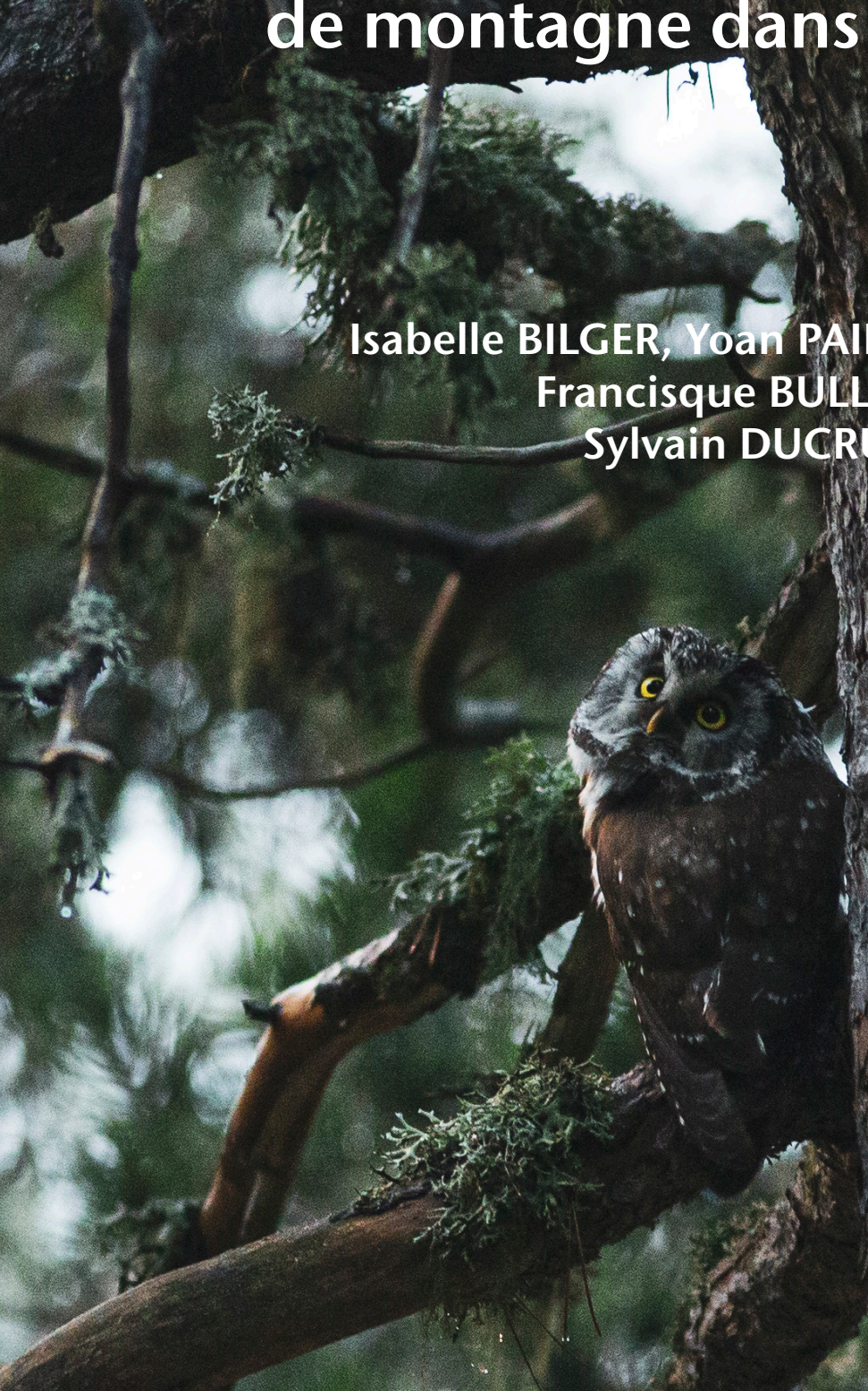


DiRECTEUR DE LA PUBLICATION / PUBLICATION DIRECTOR: Bruno David,

Président du Muséum national d'Histoire naturelle

RÉDACTEUR EN CHEF / EDITOR-IN-CHIEF: Jean-Philippe Siblet

ASSISTANTE DE RÉDACTION / ASSISTANT EDITOR: Sarah uet (naturae@mnhn.fr)

Mise EN PAGE / PAGE LAYOUT: Sarah uet

COMITÉ SCIENTIFIQUE / SCIENTIFIC BOARD:

Luc Abbadie (UPMC, Paris)

Luc Barbier (Parc naturel régional des caps et marais d'Opale, Colembert)

Aurélien Besnard (CEFE, Montpellier)

Vincent Boullet (Expert indépendant flore/végétation, Frugières-le-Pin)

Hervé Brustel (École d'ingénieurs de Purpan, Toulouse)

Patrick De Wever (MNHN, Paris)

Thierry Dutoit (UMR CNRS IMBE, Avignon)

Éric Feunteun (MNHN, Dinard)

Romain Garrouste (MNHN, Paris)

Grégoire Gautier (DRAAF Occitanie, Toulouse)

Olivier Gilg (Réserves naturelles de France, Dijon)

Frédéric Gosselin (Irstea, Nogent-sur-Vernisson)

Patrick Haffner (UMS PatriNat, Paris)

Frédéric Hendoux (MNHN, Paris)

Xavier Houard (OPIE, Guyancourt)

Isabelle Le Viol (MNHN, Concarneau)

Francis Meunier (Conservatoire d'espaces naturels - Hauts-de-France, Amiens)

Serge Muller (MNHN, Paris)

Francis Olivereau (DREAL Centre, Orléans)

Laurent Poncet (UMS PatriNat, Paris)

Nicolas Poulet (OFB, Vincennes)

Jean-Philippe Siblet (UMS PatriNat, Paris)

Laurent Tillon (ONF, Paris)

Julien Touroult (UMS PatriNat, Paris)

COUVERTURE / COVER:

La Chouette de Tengmalm Aegolius funereus (Linnaeus, 1758) est une des deux petites chouettes de montagne. Crédit photo: @samdecout.

Naturae est une revue en flux continu publiée par les Publications scientifiques du Muséum, Paris

Naturae is a fast track journal published by the Museum Science Press, Paris

Les Publications scientifiques du Muséum publient aussi / The Museum Science Press also publish:

Adansonia, Zoosystema, Anthropozoologica, European Journal of Taxonomy, Geodiversitas, Cryptogamie sous-sections Algologie, Bryologie, Mycologie, Comptes Rendus Palevol.

Diffusion - Publications scientifiques Muséum national d'Histoire naturelle

CP 41 - 57 rue Cuvier F-75231 Paris cedex 05 (France)

Tél. : 33 (0)1 40794805 / Fax: 33 (0)1 40793840

diff.pub@mnhn.fr / https://sciencepress.mnhn.fr

(c) Publications scientifiques du Muséum national d'Histoire naturelle, Paris, 2021

ISSN (électronique / electronic): 1638-9387 


\title{
Modélisation de la distribution des petites chouettes de montagne dans les Alpes du Nord françaises
}

\author{
Isabelle BILGER \\ INRAE, UR Écosystèmes forestiers, \\ Domaine des Barres, F-45290 Nogent-sur-Vernisson (France) \\ isabelle.bilger@inrae.fr \\ Yoan PAILLET \\ Université Grenoble Alpes, INRAE, LESSEM, \\ 2 rue de la Papeterie, BP76, F-38402 Saint Martin d'Hères (France) \\ yoan.paillet@inrae.fr
}

Sébastien LAGUET

Office national des Forêts, Réseau avifaune, Agence Savoie, 17 rue des Diables Bleus, F-73026 Chambéry cedex (France) sebastien.laguet@onf.fr

Baptiste DOUTAU Francisque BULLIFON Ligue pour la Protection des Oiseaux Auvergne-Rhône-Alpes, délégation territoriale Haute-Savoie, 24 rue de la Grenette, F-74370 Metz-Tessy (France) baptiste.doutau@|po.fr francisque.bulliffon@Ipo.fr

Manon CHEVALIER

Parc naturel régional du Vercors, 255 chemin des Fusillés, F-38250 Lans-en-Vercors (France) manon.chevalier@pnr-vercors.fr

Lucie DEFERNEZ Ligue pour la Protection des Oiseaux Auvergne-Rhône-Alpes, délégation territoriale Haute-Savoie, 24 rue de la Grenette, F-74370 Metz-Tessy (France) lucie.defernez@|po.fr

Sylvain DUCRUET Office national des Forêts, Réseau avifaune, Agence Savoie, 17 rue des Diables Bleus, F-73026 Chambéry cedex (France) sylvain.ducruet@onf.fr

Hervé TOURNIER

Parc naturel régional du Vercors, 255 chemin des Fusillés, F-38250 Lans-en-Vercors (France) herve.tournier@pnr-vercors.fr

Frédéric ARCHAUX

INRAE, UR Écosystèmes forestiers, Domaine des Barres, F-45290 Nogent-sur-Vernisson (France) frederic.archaux@inrae.fr 
MOTS CLÉS

Prédiction spatiale, probabilité de présence, modèles,

Aegolius funereus, Glaucidium passerinum Rhône-Alpes, conservation de l'habitat.
Bilger I., Paillet Y., Laguet S., Doutau B., Bullifon F., Chevalier M., Defernez L., Ducruet S., Tournier H. \& Archaux F. 2021. - Modélisation de la distribution des petites chouettes de montagne dans les Alpes du Nord françaises. Naturae 2021 (13): 167-181. https://doi.org/10.5852/naturae2021a13

\section{RÉSUMÉ}

Les petites chouettes de montagne (Chouette de Tengmalm, Aegolius funereus (Linnaeus, 1758) et Chevêchette d'Europe, Glaucidium passerinum (Linnaeus, 1758)) sont représentées en France par quelques centaines à quelques milliers de couples. Elles font l'objet d'une attention particulière au regard de leur rareté et en tant qu'espèces emblématiques des écosystèmes forestiers de montagne, qui sont particulièrement affectés par les changements globaux. Améliorer la connaissance de leur distribution, notamment dans les Alpes du Nord, est une nécessité pour préserver les milieux qui constituent leur habitat préférentiel. Issue d'une collaboration entre l'INRAE, l'Office national des Forêts et la Ligue pour la Protection des Oiseaux, nous présentons une approche par modélisation dans laquelle des données d'occurrence des deux espèces sur la période 2007-2014 ont été reliées à six variables environnementales potentiellement explicatives pour prédire leur répartition. Les résultats montrent que la distribution des deux espèces est principalement liée d'une part à l'altitude, facteur déterminant au plan régional, et d'autre part au type d'occupation du sol, meilleur prédicteur à l'échelle locale, alors que les autres facteurs (topographie, proximité de lisières) sont moins explicatifs. Ces prédictions ont été utiles aux naturalistes de terrain, qui ont pu utiliser ces données pour mieux structurer leurs campagnes d'observations. Il serait désormais intéressant d'étendre la démarche à d'autres zones biogéographiques et d'intégrer à la modélisation des données permettant d'évaluer les effets de la gestion forestière sur ces espèces.

\begin{abstract}
Distribution modelling of small mountain owls in the Northern French Alps.

Small mountain owls (the Tengmalm Owl, Aegolius funereus (Linnaeus, 1758) and the Eurasian Pygmy Owl, Glaucidium passerinum (Linnaeus, 1758)) comprise a few hundred to a few thousand couples in France. Their conservation is at stake regarding their rarity, especially with respect to global change issues. However, their distributions remind little known, especially in the Northern Alps, where presence data are available. Through a collaboration between INRAE, la Ligue pour la Protection des Oiseaux et l'Office national des Forêts, we used occurrence data (2007-2014) to model the distribution of the two species based on six environmental variables that potentially affect their distribution. We showed that the distribution of the two species is mainly driven by elevation and land-use type, while other factors (topography, distance to edges) are less explanatory. These predictions have been used by field naturalists, who structured their field campaigns based on the prediction maps. To continue this research, it seems interesting to extend the approach to other biogeographic contexts and integrate variables that would allow assessing the effects on forest management on these species to the models.
\end{abstract}

KEY WORDS Spatial prediction, probability of presence,

Aegolius funereus, Glaucidium passerinum, Rhône-Alpes, habitat conservation.

\section{INTRODUCTION}

Le cadre de gestion multifonctionnelle des espaces naturels, notamment forestiers, vise à obtenir des compromis entre leurs différentes fonctions: production de biens et services d'une part et protection des milieux et des espèces d'autre part. Pour combiner ces objectifs, un préalable est d'identifier pour un territoire donné, les enjeux propres à chacune des fonctions considérées, de spatialiser ces enjeux et de les hiérarchiser dans les différentes zones (Barthod 2015). Ainsi, la prise en compte de la biodiversité dans les politiques de gestion des milieux naturels nécessite une bonne connaissance de l'état et de la dynamique des populations d'espèces cibles, en particulier des espèces rares et menacées dont la préservation est souvent considérée comme un enjeu prioritaire.

C'est notamment le cas des petites chouettes de montagne, Chouette de Tengmalm, Aegolius funereus (Linnaeus, 1758) et Chevêchette d'Europe, Glaucidium passerinum (Linnaeus, 1758) (Fig. 1), représentées en France par quelques centaines à quelques milliers de couples. Étroitement associées aux forêts de montagne dans le sud de l'Europe, elles font l'objet d'une attention particulière au plan national et international au regard de leur rareté et en tant qu'espèces emblématiques des écosystèmes forestiers de montagne, qui sont particulièrement sensibles aux changements globaux. Elles figurent toutes le deux à l'Annexe I de la Directive oiseaux et à l'Annexe II de la Convention sur le Commerce international des Espèces (CITES). La Chevêchette d'Europe est quant à elle inscrite sur la liste rouge des espèces menacées de France dans la catégorie "quasi menacée» (UICN France et al. 2016). Ces deux espèces ont des exigences fortes tant à l'échelle de la mosaïque paysagère - leur territoire englobant à la fois des milieux ouverts (clairières, prairies) et des peuplements fermés - qu'à l'échelle de la parcelle, avec des 

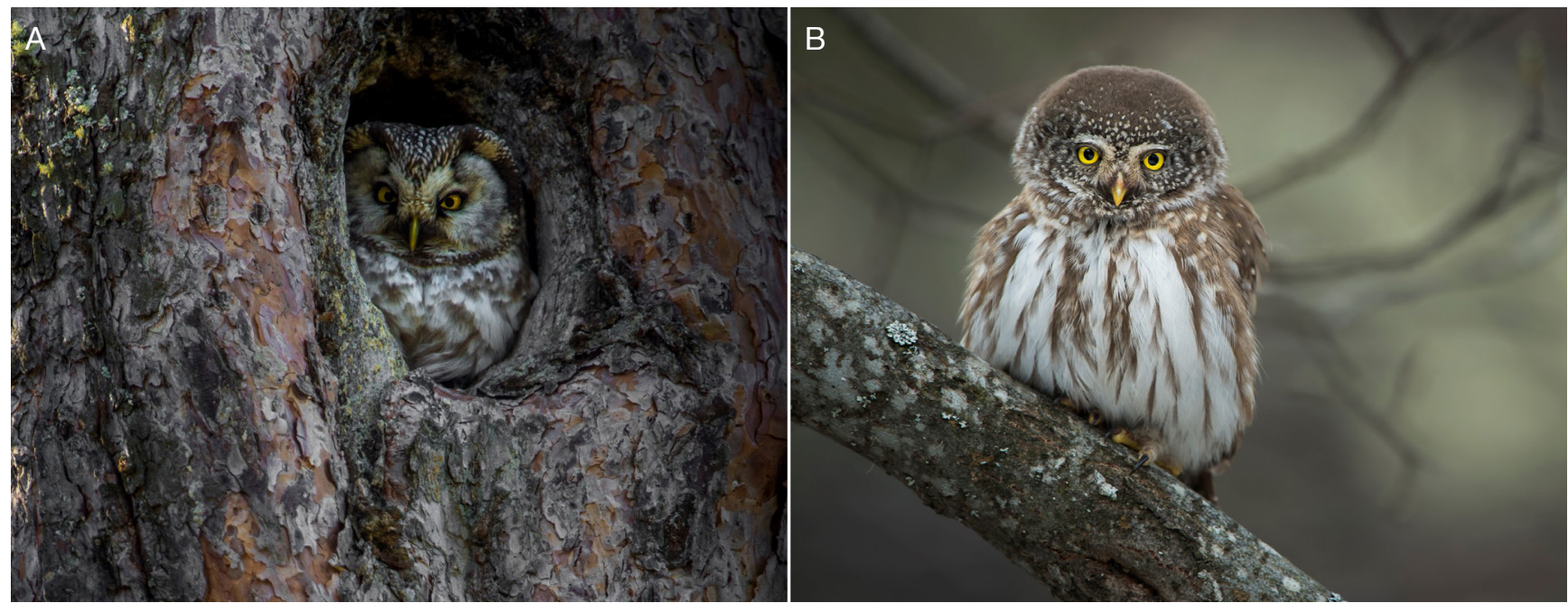

FIG. 1. - Chouette de Tengmalm, Aegolius funereus (Linnaeus, 1758) (A) et Chevêchette d'Europe, Glaucidium passerinum (Linnaeus, 1758) (B). Crédit photo: S. De Danieli.

préférences marquées vis-à-vis de la composition et de la structure des peuplements (présence d'arbres matures voire sénescents, de sapin, d'épicéa ou de hêtre en particulier). Ces éléments jouent un rôle déterminant dans la structuration des domaines vitaux des deux espèces car ils fournissent à la fois des sites de chasse en milieux ouverts intra-forestiers et en lisière, des proies, notamment des rongeurs, et des sites de nidification dans les cavités d'arbres (Brambilla et al. 2013; Barbaro et al. 2016).

Depuis 2008, sous l'égide de la Ligue pour la Protection des Oiseaux (LPO) et l'Office national des Forêts (ONF), le réseau national «Petites chouettes de montagne» réunit naturalistes et observateurs et s'est structuré de manière à intensifier et améliorer le suivi de ces oiseaux. Une de ses premières missions a consisté à rassembler les données d'observations au plan national pour établir des synthèses annuelles. Sur les sept départements de la région Rhône-Alpes, on dénombre, lors des bonnes années, de 90 à 230 sites (individus ou couples) pour la Chouette de Tengmalm, soit en moyenne $32 \%$ de l'effectif national estimé, et de 130 à 200 sites pour la Chevêchette d'Europe, soit $50 \%$ de l'effectif national estimé (Ligue pour la Protection des Oiseaux 2019). Aux côtés de sites connus pour être fréquentés régulièrement depuis plusieurs années par ces espèces, de nouvelles présences sont régulièrement documentées, y compris dans des zones inhabituelles ou atypiques, par exemple pour la Chevêchette d'Europe en Haute-Marne et Rhône en 2017, en Meurthe-et-Moselle en 2018 et pour la Chouette de Tengmalm, en pinède noire dans les Causses (Guérin B, comm. pers). De même, la progression depuis 2016 du nombre d'individus chanteurs détectés dans l'Aude, l'Ariège et le Limousin témoignent d'une expansion géographique vers le sud-ouest du Massif central et les Pyrénées. Ces nouvelles découvertes justifient, en plus de poursuivre les campagnes de prospections dans des secteurs où les espèces ont déjà été repérées, de couvrir de nouveaux secteurs pour mieux cerner les milieux et facteurs propices à ces espèces. Or, la forte fluctuation interannuelle des effectifs de ces deux espèces, associée à leur discrétion, imposent de multiplier les sorties de terrain durant une saison et pendant plusieurs années pour améliorer la fiabilité des données d'occurrence. Cependant, une telle pression d'observation ne peut être réalisée partout pour des raisons techniques et budgétaires. Ces contraintes incitent les membres du réseau à adopter une démarche plus structurée afin de renforcer la pression d'échantillonnage tout en optimisant ces efforts. Cette stratégie inclut à la fois des suivis avec un protocole standardisé sur des zones étudiées tous les ans sur de longues durées (Laguet \& Besnard 2019) et en parallèle, des prospections ciblées sur des secteurs non prospectés par le passé mais considérés a priori propices à ces espèces.

La présente étude vise à établir une carte de probabilité de présence de chacune des deux chouettes à l'échelle de l'ex-région Rhône-Alpes sur la base de données environnementales déjà disponibles. Cette approche est basée sur la modélisation de la distribution spatiale d'espèces à grande échelle, à partir des données recueillies depuis 2007 et d'informations cartographiques sur l'occupation du sol et autres variables environnementales. Ces cartes de prédiction ont été utilisées en 2017 et 2018 par des gestionnaires d'espaces naturels comme outil pour orienter leur recensement de populations des chouettes. Leur témoignage apporte une vérification pratique de la pertinence de cette modélisation.

\section{MATÉRIEL ET MÉTHODES}

\section{ZONE D'ÉTUDE}

La zone d'étude retenue pour la modélisation couvre le territoire des huit départements de l'ancienne région administrative RhôneAlpes (soit $3260 \mathrm{~km}^{2}$ ). Elle inclut, comme zones de montagne, le sud du Massif jurassien (Ain), les contreforts est du Massif central (Ardèche, Loire et Rhône) et les parties septentrionales et centrales du Massif alpin (Isère, Drôme, Savoie et Haute-Savoie). Par simplification, l'étendue de la zone d'extrapolation définie pour la modélisation n'a pas été limitée aux tranches altitudinales où les espèces sont présentes habituellement. 
TABLEAU 1. - Distribution des données de présence et absence par département pour la Chevêchette d'Europe Glaucidium passerinum (Linnaeus, 1758) et la Chouette de Tengmalm Aegolius funereus (Linnaeus, 1758).

\begin{tabular}{|c|c|c|c|c|c|c|}
\hline \multirow[b]{2}{*}{ Départements } & \multicolumn{3}{|c|}{ Chevêchette d'Europe } & \multicolumn{3}{|c|}{ Chouette de Tengmalm } \\
\hline & Présence & Absence & Total & Présence & Absence & Total \\
\hline Ain & 45 & 48 & 93 & 12 & 11 & 23 \\
\hline Drôme & 45 & 5 & 50 & 31 & 2 & 33 \\
\hline Isère & 789 & 589 & 1378 & 286 & 264 & 550 \\
\hline Savoie & 182 & 95 & 277 & 115 & 40 & 155 \\
\hline Haute-Savoie & 255 & 592 & 847 & 118 & 290 & 408 \\
\hline Total & 1316 & 1329 & 2645 & 562 & 607 & 1169 \\
\hline
\end{tabular}

La méthode décrite ici s’inspire de travaux de modélisation précédemment entrepris pour la Chevêchette d'Europe (Redon 2012) et deux espèces de chauve-souris sur une zone restreinte de l'Isère et de la Drôme (Le Roux et al. 2017).

\section{DONNÉES D'OBSERVATION}

Les données d'observation des deux espèces de chouettes ont été obtenues par extraction de différentes bases de données gérées par les associations LPO locales et l'ONF. Elles correspondent à des informations collectées par contacts directs visuels ou auditifs, établis par hasard ou dans le cadre d'opérations de recherche généralement non standardisées. Après vérification et harmonisation de toutes les données et élimination de celles ayant une géolocalisation trop imprécise (i.e., > $100 \mathrm{~m}$, sur la base des déclarations des observateurs), 3814 données d'observation de présence et d'absence recueillies entre 2007 et 2014, ont été retenues dont 2645 pour la Chevêchette d'Europe (dont 1316 présences et 1329 absences) et 1169 pour la Chouette de Tengmalm (dont 562 présences et 607 absences). Toutes les données antérieures à 2007 ont été écartées car elles étaient trop sporadiques et leur localisation généralement peu précise. Les occurrences retenues sont inégalement réparties dans cinq des huit départements (Ain, Drôme, Isère, Savoie et Haute-Savoie, Tableau 1); elles se concentrent sur 250 communes environ, situées entre 720 et 1850 mètres d'altitude pour la Chouette de Tengmalm et 730 et 2200 mètres pour la Chevêchette d'Europe (Fig. 2).

Pour chacune des espèces étudiées séparément, nous avons généré à partir de ces données d'observation précisément géolocalisées un fichier raster binaire $(0 / 1)$ avec une résolution de 30 mètres calée sur le modèle numérique de terrain (MNT, BD ALTI ${ }^{\oplus} 30 \mathrm{~m}$ de l'Institut géographique national). Dans le cas où plusieurs données d'observation étaient associées à un même pixel (présences ou absence relevées sur un même site mais à des dates différentes: années, mois ou jours successifs), une seule valeur était attribuée à ce pixel: si au moins une présence était mentionnée, alors la valeur 1 était attribuée au pixel, dans le cas contraire, la valeur 0 était attribuée. Le fichier raster obtenu comportait au final 2487 pixels (pour 2645 observations) renseignés pour la Chevêchette d'Europe (1258 pixels en présence et 1229 pixels en absence) et 1074 pixels (pour 1139 observations) pour la Chouette de Tengmalm ( 550 pixels en présence et 524 pixels en absence).

\section{VARIABLES ENVIRONNEMENTALES}

Les variables environnementales ont été choisies en se focalisant a priori sur des paramètres susceptibles d'avoir une influence sur la distribution des deux espèces, donc en tenant compte des caractéristiques écologiques des espèces. Ces variables devaient par ailleurs être facilement accessibles et disponibles sous forme standardisée sur l'ensemble de la région. Au total six variables spatialisées ont été retenues pour les deux espèces, afin de caractériser en chaque point de la zone d'étude:

- la topographie issue du modèle numérique de terrain et caractérisée par l'altitude et un indice topographique semiquantitatif dérivé de l'altitude (1. Vallée; 2. Plaine; 3. Versant; 4. Crête);

- les types de peuplements forestiers et d'occupation biophysique des sols caractérisés par le type d'occupation des sols, construit en assemblant au sein d'une même couche géographique les types de peuplements forestiers de la $\mathrm{BD}$ FORET $^{\oplus} \mathrm{V} 2$ de l'IGN pour les zones forestières et les types de milieux issus de CORINE Land Cover 2006 pour les zones hors forêts. Un regroupement en huit classes a ainsi été établi de façon à différencier, au sein des zones boisées, les types de peuplements forestiers et, hors zone boisée, les milieux semi-naturels ouverts, les milieux agricoles cultivés, les zones aquatiques et les zones ayant un fort degré d'anthropisation (Tableau 2);

- la proximité de milieux ouverts semi-naturels caractérisée par la distance à la plus proche prairie, calculée à partir de CORINE Land Cover 2006;

- un degré d'anthropisation grossièrement évalué par la distance à la zone urbanisée la plus proche et la distance à la route la plus proche, calculées à partir de la $\mathrm{BD} \mathrm{TOPO}^{\circ}$ de l'IGN.

Chacune de ces six variables spatiales ont été traitées de manière à obtenir des couches raster complètes sur l'ensemble de la zone d'étude avec une résolution de 30 mètres.

\section{MODÉLISATION DE LA DISTRIBUTION DES ESPÈCES:} UTILISATION ET PARAMÉTRAGE DE L'OUTIL BIOMOD2 L'utilisation en écologie de modèles prédictifs de distribution spatiale d'espèces («Species Distribution Models» en anglais) s'est considérablement développée au cours des quinze dernières années et permet notamment de guider des programmes de gestion et de conservation d'espèces rares ou menacées. Le principe de ces modèles est d'estimer une probabilité de présence en fonction de diverses informations environnementales sur 
A

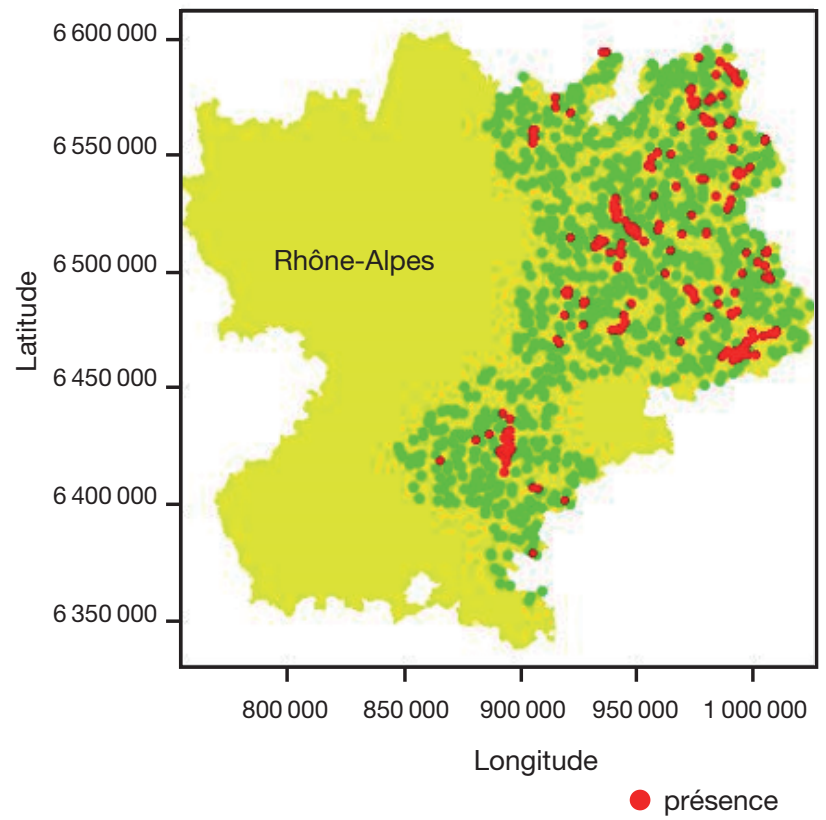

B

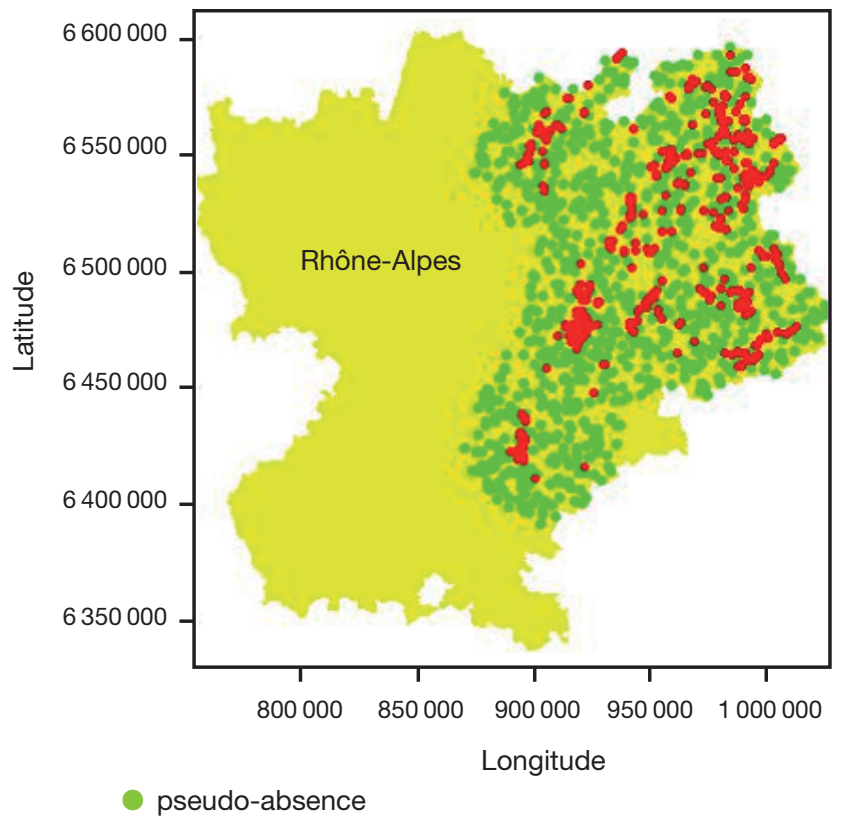

FIG. 2. - Répartition des points utilisés pour la modélisation: présences réelles et 1000 pseudo-absences tirées au sort dans un rayon maximal de 20 km (min. 60 m). A, Chouette de Tengmalm, Aegolius funereus (Linnaeus, 1758); B, Chevêchette d'Europe, Glaucidium passerinum (Linnaeus, 1758). Latitude et Longitude (système Lambert 93 en mètres).

TABLEAU 2. - Huit classes obtenues par le regroupement des types de peuplement (BD Forêt V2) et Corine Land Cover 2006.

\begin{tabular}{|c|c|}
\hline Code & Type de peuplement \\
\hline 1 & $\begin{array}{l}\text { Cours et voies d'eau, plans d'eau, marais intérieurs, terres arables hors périmètre d'irrigation, vignobles et petits fruits, } \\
\text { oliveraies, systèmes culturaux et parcellaires complexes }\end{array}$ \\
\hline 2 & $\begin{array}{l}\text { Landes et broussailles, forêt et végétation arbustive en mutation, formations herbacées, végétation sclérophylle, forêt fermée } \\
\text { ou ouverte sans couvert arboré }\end{array}$ \\
\hline 3 & Forêts mélangées, forêt fermée à mélange de feuillus prépondérants et conifères \\
\hline 4 & Forêt fermée de sapin ou d'épicéa \\
\hline 5 & Forêt fermée de conifères purs, de mélange de conifères ou mélange de conifères prépondérants et feuillus \\
\hline 6 & Forêt fermée de feuillus purs ou de mélange de feuillus \\
\hline 7 & Prairies, pelouses, pâturages naturels et végétation clairsemée \\
\hline 8 & Forêt ouverte de feuillus, conifères ou mélange feuillus-conifères \\
\hline
\end{tabular}

les points connus de présence d'une espèce (et éventuellement d'absence) puis d'extrapoler cette probabilité en tout point de l'espace de la zone d'étude à partir de ces mêmes données environnementales: cette probabilité fournit une indication sur les zones potentiellement favorables aux espèces étudiées. Ces modèles permettent ainsi de prédire la répartition potentielle d'espèces rares ou difficiles à observer dans des secteurs non encore ou sporadiquement couverts par les prospections. Le recours à ces modèles paraissait donc approprié pour identifier de nouveaux sites potentiels de présence en Rhône-Alpes de ces deux espèces de chouettes discrètes et difficiles à détecter.

La modélisation de la distribution des deux chouettes a été réalisée sous le logiciel $\mathrm{R}$ version 3.2.4 (R Core Team 2015) en utilisant la méthode multi-modélisation («Ensemble modeling") via la plateforme BIOMOD (Thuiller et al. 2009). Ce package Biomod 2 permet de tester en parallèle différents algorithmes de modélisation et de comparer leurs résultats afin de choisir le plus pertinent. Sept types de modèles statistiques sont implémentés dans Biomod2: Artificial Neural Networks (ANN), Classification Tree Analysis (CTA), Flexible Discriminant Analysis (FDA), Generalised Additive Models (GAM), Generalised Boosted Models (GBM), Generalised Linear Models (GLM) et Random Forests (RF). Le huitième type de modèle Maximum Entropy (MaxEnt) n'a pas pu être utilisé avec les options retenues pour la phase de calibration. Lorsque les données d'absence sont inexistantes ou non fiables, par exemple seulement sur certains secteurs ou milieux, Biomod2 permet également de générer aléatoirement des données de "pseudo-absence» selon différentes stratégies paramétrables. C'est cette méthode qui a été utilisée ici.

Parmi les données digitalisées lors de l'étape précédente, $80 \%$ choisies aléatoirement ont été utilisées pour calibrer 
TABLEAU 3. - Indice pour juger la qualité prédictive des modèles (selon Thuiller et al. 2010)

\begin{tabular}{llll}
\hline $\begin{array}{c}\text { Qualité } \\
\text { prédictive }\end{array}$ & \multicolumn{1}{c}{ TSS } & $\begin{array}{c}\text { Indice de } \\
\text { Kappa }\end{array}$ & \multicolumn{1}{c}{ ROC } \\
\hline Faible & 0,2 à 0,4 & 0,2 à 0,4 & 0,6 à 0,7 \\
Correcte & 0,4 à 0,6 & 0,4 à 0,6 & 0,7 à 0,8 \\
Bonne & 0,6 à 0,8 & 0,6 à 0,8 & 0,8 à 0,9 \\
Excellente & 0,8 à 1 & 0,8 à 1 & 0,9 à 1 \\
\hline
\end{tabular}

les modèles et les $20 \%$ restantes, pour la validation interne dite croisée. Trois paramètres statistiques, calculés sur les données de validation pour chaque modélisation, ont été utilisés pour évaluer la précision et la pertinence des modèles (Tableau 3) :

- la statistique de compétence vraie ("True Skill Statistic», TSS) est un indice d'ajustement de modèle par rapport à des données d'occurrence. Le TSS varie de -1 à 1 ; plus il est proche de 1, meilleur est l'accord (1 indique l'accord parfait);

- le Kappa de Cohen qui mesure l'accord entre les données modélisées et les données de validation. Comme le TSS, le Kappa varie de -1 à 1 ; plus il est proche de 1 , meilleur est l'accord;

- la courbe dite "ROC" (pour "caractéristique du fonctionnement du récepteur", en anglais) évalue la performance d'une classification en comparant le taux de vrais positifs, c'est-à-dire la fraction des présences effectivement détectées, et des faux positifs correspondant à la fraction des absences incorrectement classées en présences. Les résultats sont visualisés sous forme d'une courbe qui représente les taux de vrais positifs en fonction des taux de faux positifs, le but étant de maximiser les premiers tout en minimisant les seconds.

Pour chaque modélisation, le package Biomod 2 calcule également la contribution des différentes variables environnementales à la prédiction globale des probabilités de présence sur la zone d'étude.

Enfin, pour évaluer de manière indépendante la pertinence des cartes prédictives de présence issues de ces modélisations, nous avons confronté les résultats avec les données de l'Atlas des oiseaux de France métropolitaine (Muller \& Issa 2015) qui renseigne sur la présence de l'espèce pour chaque maille de $10 \times 10 \mathrm{~km}$ selon un indice en trois catégories (reproduction certaine, probable ou possible) en synthétisant les informations collectées de 2005 à 2015. Cette comparaison est purement qualitative, car la maille de l'Atlas $(10 \times 10 \mathrm{~km})$ et celle de la modélisation $(30 \times 30 \mathrm{~m})$ ne permettaient pas de comparaison directe des résultats, ni de test statistique approprié.

\section{RÉSULTATS}

Distribution DES PETITES CHOUETTES DE MONTAGNE À L'ÉCHELLE DE L'EX-RÉGION RHÔNE-ALPES Paramètres de modélisation, qualité des prédictions et influence des variables environnementales

Les premières analyses avec l'ensemble des données de présence et d'absence ont fourni des prédictions exagérément optimistes voire fantaisistes. Nous avons donc choisi de n'utiliser comme données de calibration que les relevés de présence, soit 1258 points (pixels) pour la Chevêchette d'Europe et 550 pour la Chouette de Tengmalm. À ces présences, ont été associées 1000 pseudo-absences générées selon la stratégie «Disk», c’està-dire dans un rayon autour de chaque point de présence avec comme paramètres une distance minimale de $60 \mathrm{~m}$ et une distance maximale de $20 \mathrm{~km}$ (Fig. 2). Nous ne présentons ici que les résultats issus des modélisations avec ces paramètres. Les autres paramétrages testés, tels que la stratégie "aléatoire» ou des distances minimales de $100 \mathrm{~m}$ et maximales de $25 \mathrm{ou}$ $30 \mathrm{~km}$, ont donné des résultats moins probants.

La comparaison des valeurs des paramètres statistiques TSS, kappa et ROC révèle que les deux meilleurs modèles sont systématiquement, et pour les deux espèces, le Random Forest suivi du Generalised Boosted Model selon les différentes combinaisons testées pour générer les pseudo-absences (Figs 3, 4).

\section{Influence des variables environnementales}

Pour les deux espèces, les variables environnementales qui ont le plus de poids dans les prédictions sont l'altitude et le type d'occupation du sol, à savoir soit le type de peuplements forestiers, soit la présence de milieux semi-naturel ou cultivé (Tableau 4).

Pour la Chevêchette d'Europe, l'altitude contribue pour $38 \%$ à la probabilité de présence et l'occupation du sol pour environ $35 \%$. Les variables de distance à la plus proche prairie, zone urbanisée et route contribuent chacune à au moins $8 \%$ alors que la variable topographique intervient peu (2\%). Les milieux les plus favorables sont les peuplements fermés de conifères ou de mélange conifères/feuillus et les peuplements ouverts, situés à une altitude supérieure à $800 \mathrm{~m}$.

Pour la Chouette de Tengmalm, l'altitude contribue pour $35 \%$ et l'occupation du sol pour $32 \%$. Les variables de distance à la plus proche zone urbanisée et route ont un poids légèrement plus fort $(11 \%)$ que pour la Chevêchette d'Europe alors que les deux variables «distance à la plus proche prairie» et "variable topographique» ont la même importance, soit respectivement $8 \%$ et $2 \%$. Les milieux favorables sont les peuplements fermés, quelle que soit la composition dominante (coniferes, feuillus ou mixte), situés à plus de 900 m d'altitude.

\section{Seuillage des prédictions}

La cartographie obtenue pour chacune des espèces fournit, pour chaque pixel de la zone d'étude, une valeur correspondant à la probabilité d'occurrence (en \%) de l'espèce, calculée selon le ou les deux modèles statistiques considérés comme les meilleurs. En fixant un seuil à cette probabilité, il est possible d'obtenir une réponse binaire, c'est-à-dire de définir si la probabilité correspond à une présence ou à une absence et d'obtenir une carte des habitats favorables. Cette façon de faire permet une représentation cartographique plus aisée pour orienter des prospections. En fixant un seuil de probabilité de présence de $80 \%$ sur les meilleurs modèles pour représenter les "habitats favorables", on obtient pour les deux espèces, des cartes dans lesquelles les habitats potentiellement favorables couvrent 6 à $7 \%$ du territoire de la région soit 2830 à $3260 \mathrm{~km}^{2}$ (Fig. 5). 


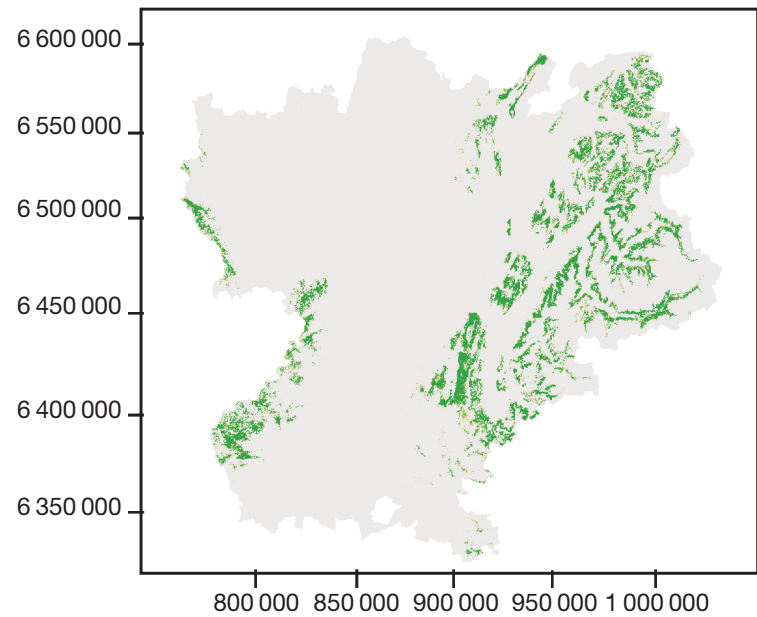

RF (TSS=0,982, Kappa=0,929, ROC $=1$ )

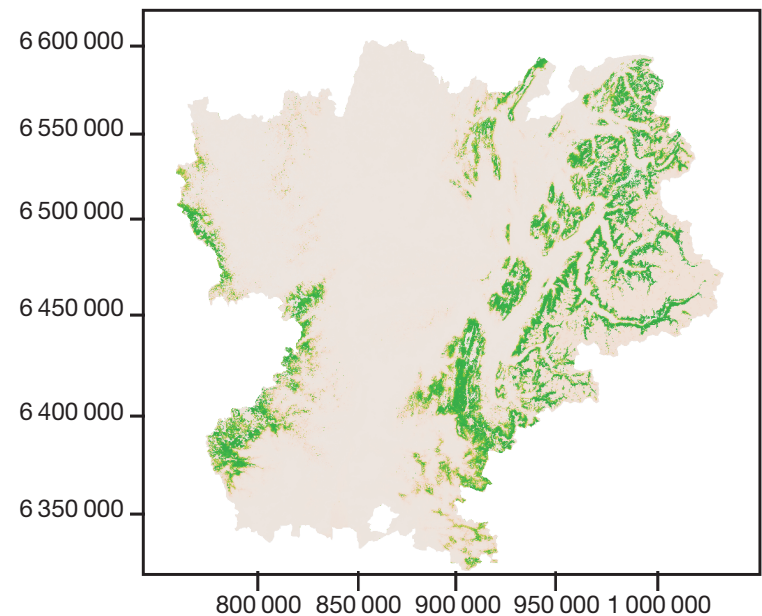

ANN (TSS=0,766, Kappa $=0,654, \mathrm{ROC}=0,917$ )

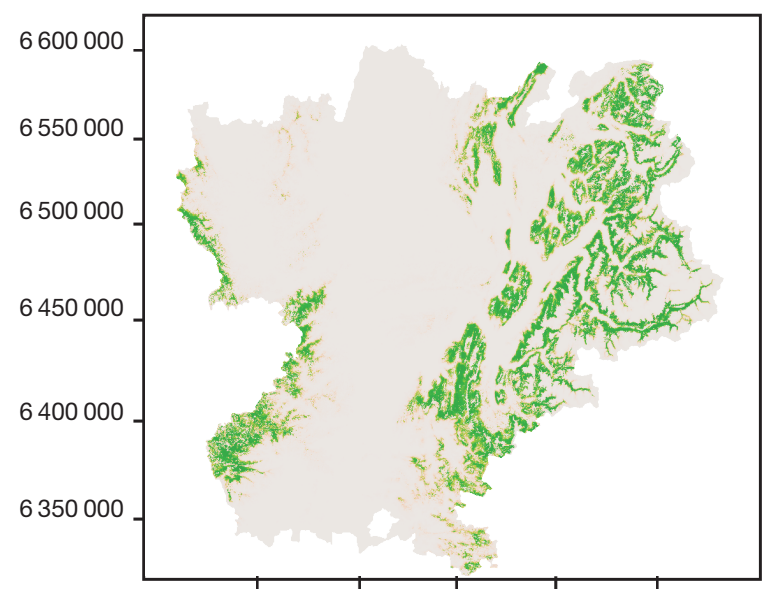

8000008500009000009500001000000

GLM (TSS=0,727, Kappa=0,62, ROC=0,916)

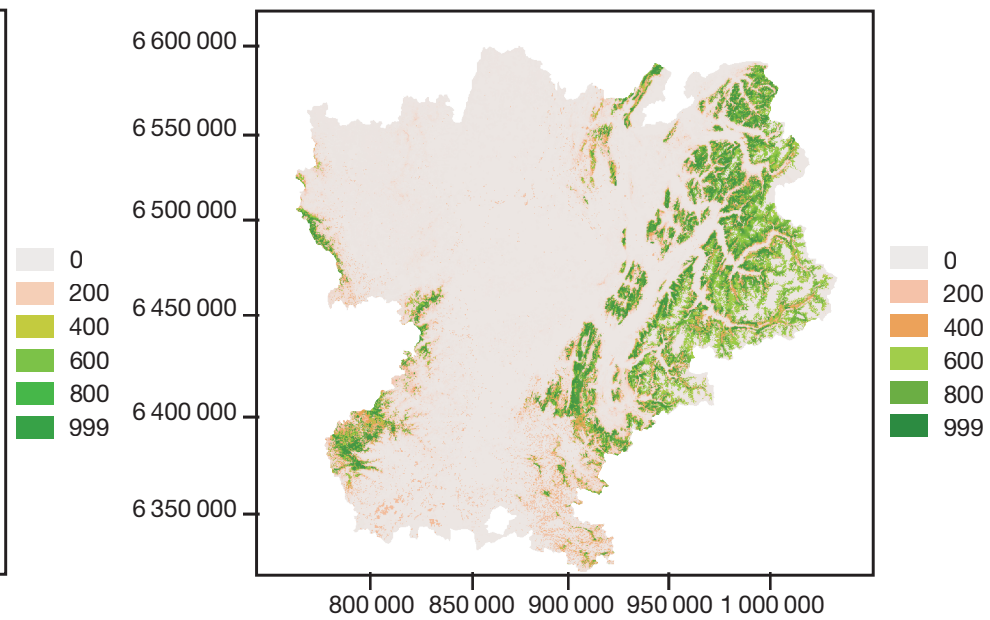

GBM (TSS=0,809, Kappa $=0,704, \mathrm{ROC}=0,954$ )

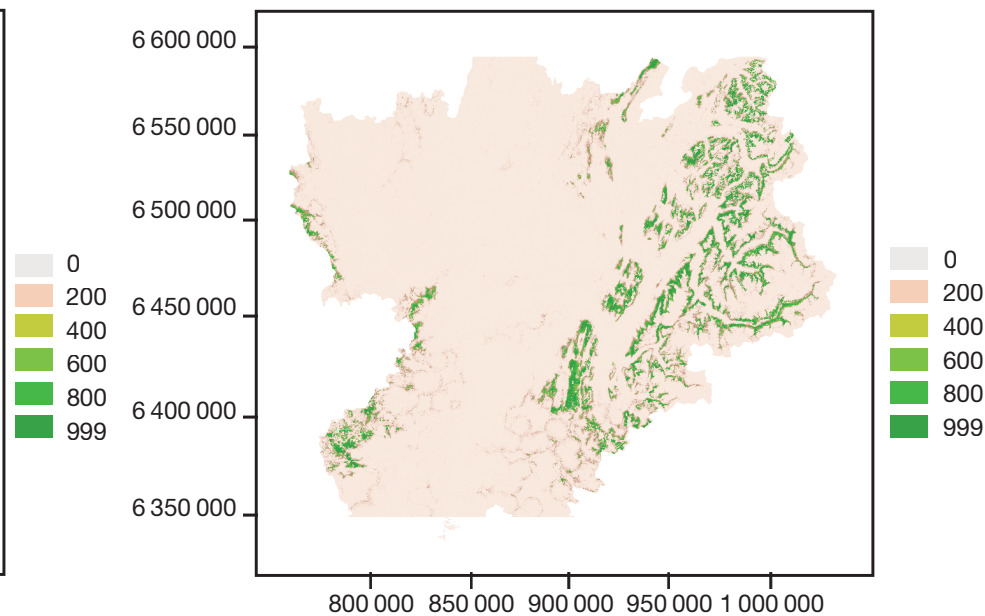

FDA (TSS $=0,734$, Kappa $=0,661, \mathrm{ROC}=0,917$ )

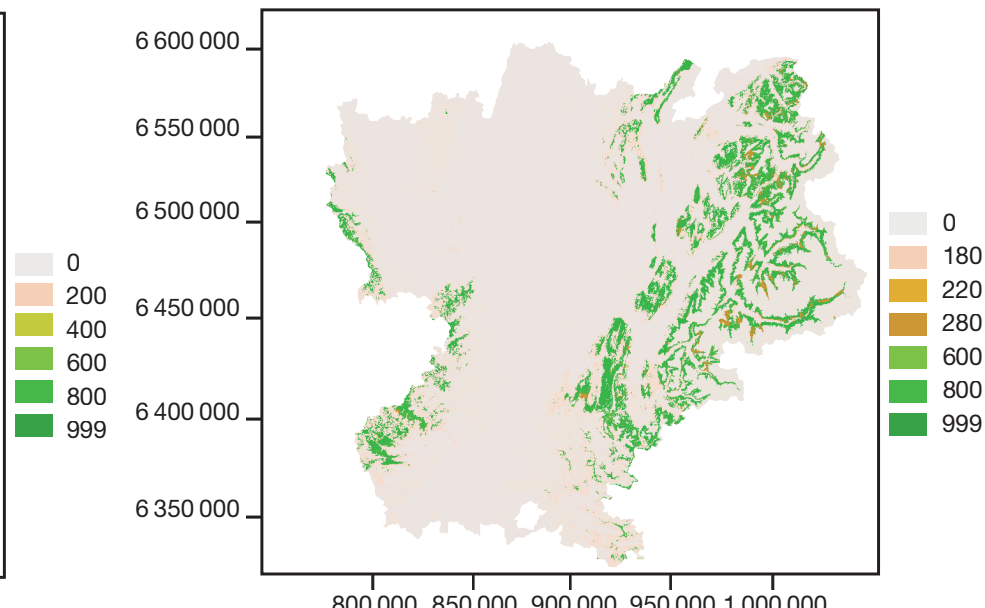

CTA (TSS $=0,748$, Kappa $=0,619, \mathrm{ROC}=0,888$ )

FIG. 3. - Cartes de prédiction pour la Chouette de Tengmalm Aegolius funereus (Linnaeus, 1758) en Rhône-Alpes. Les six premiers modèles testés dans Biomod2 sont classés par ordre de qualité de prédiction décroissante (indices TSS, Kappa et ROC). Les modélisations utilisent 524 points (pixels) de présences réelles et 1000 pseudo-absences générées selon la stratégie «disk» avec distance comprise entre $60 \mathrm{~m}$ et $20 \mathrm{~km}$. Abréviations: ANN, artificial neural networks; CTA, classification tree analysis; FDA, flexible discriminant analysis; GBM, generalised boosted models; GLM, generalised linear models ; RF, random forests. Les valeurs de TSS (True Skill Statistic), de Kappa et de ROC (caractéristique du fonctionnement du récepteur en anglais) indiquent la performance des modèles: plus la valeur est proche de 1, meilleur est le modèle prédictif. En abscisse, la longitude et en ordonnée, la latitude (système de référence Lambert 93 en mètres). 


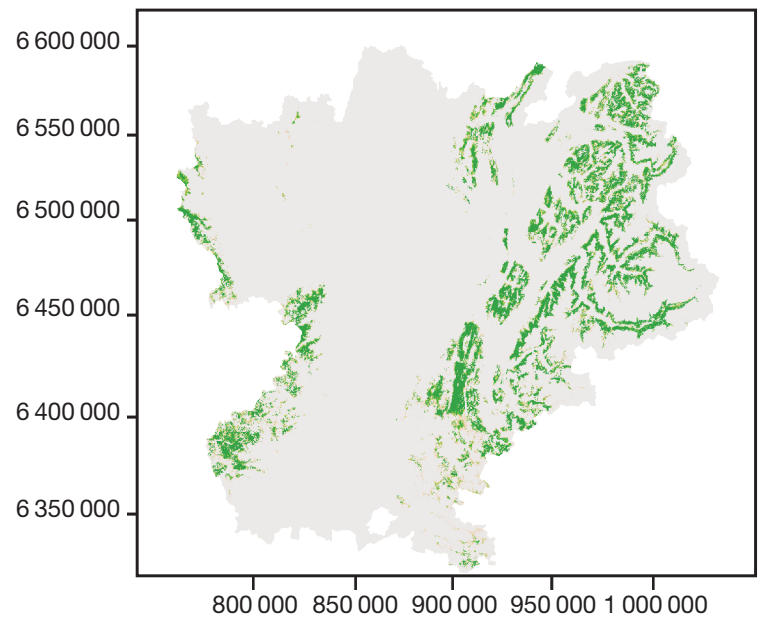

RF (TSS=0,955, Kappa=0,959, ROC=0.999)

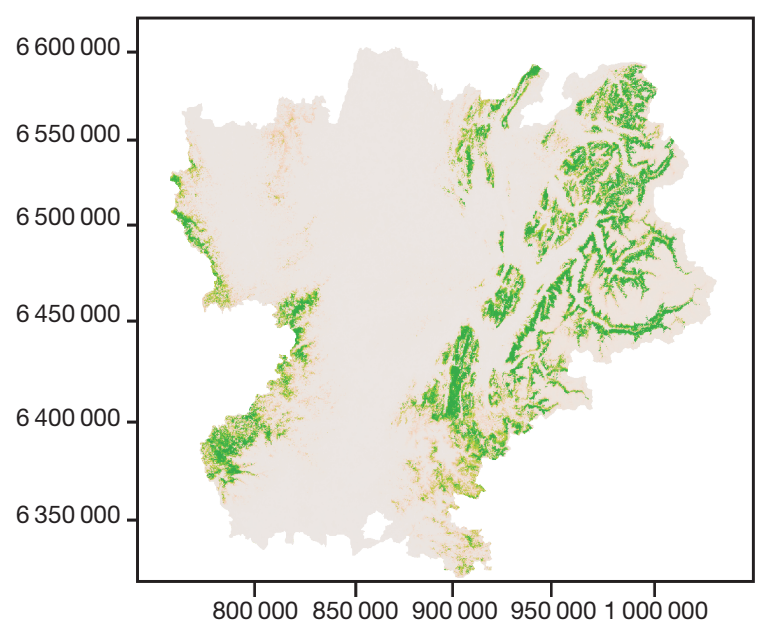

ANN (TSS=0,75, Kappa $=0,724, \mathrm{ROC}=0,929)$

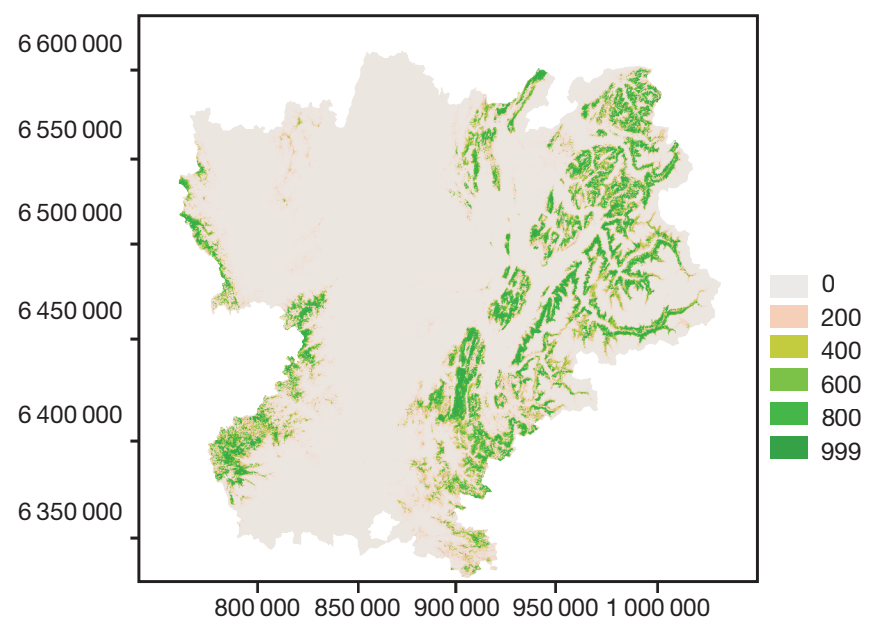

GLM (TSS=0,742, Kappa $=0,721, \mathrm{ROC}=0,93)$

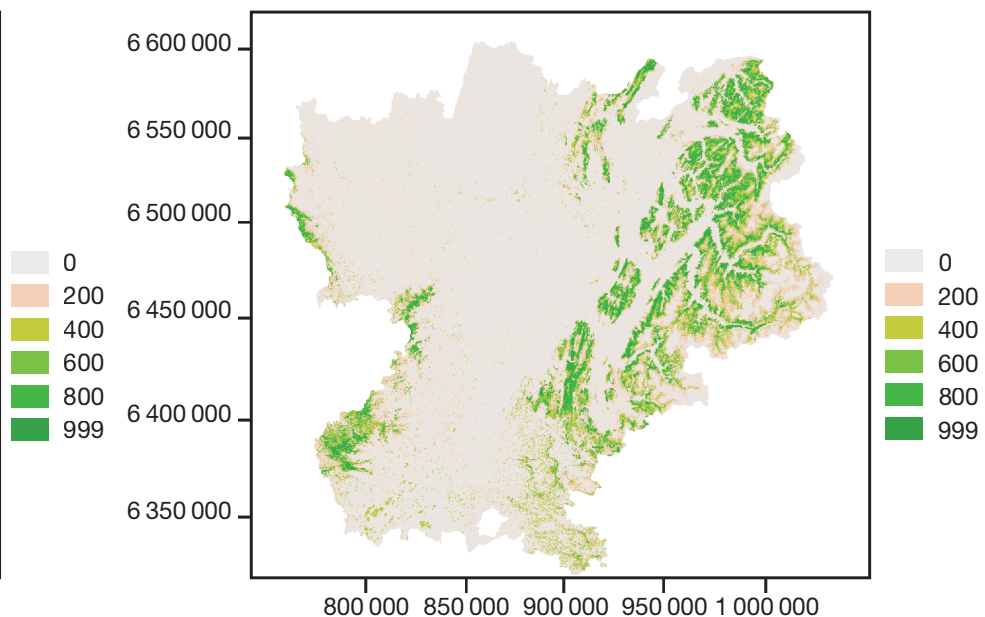

GBM (TSS=0,786, Kappa $=0,774, \mathrm{ROC}=0,953)$

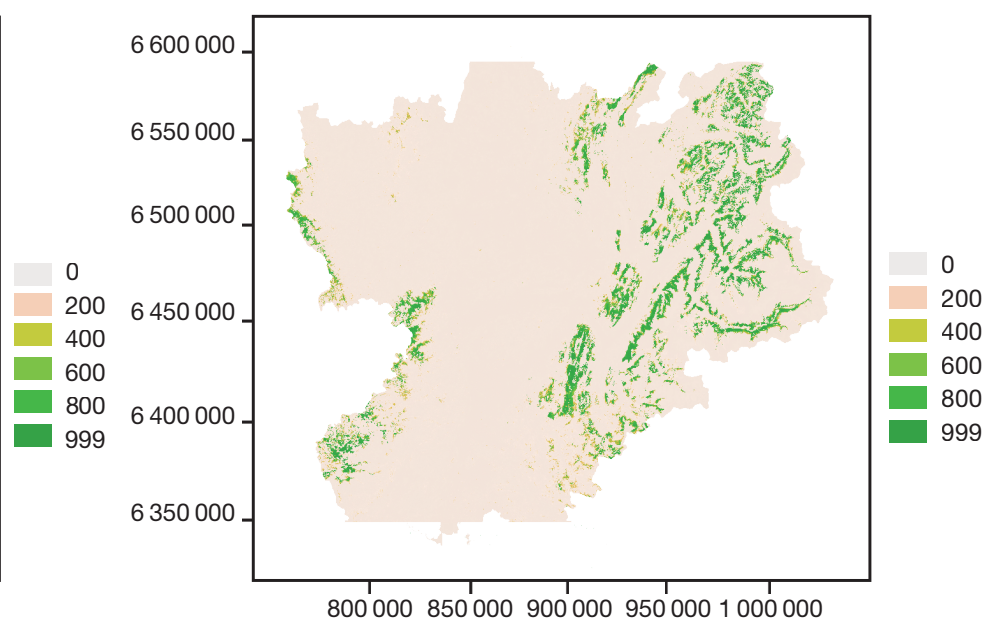

FDA (TSS=0,747, Kappa $=0,719, \mathrm{ROC}=0,927)$

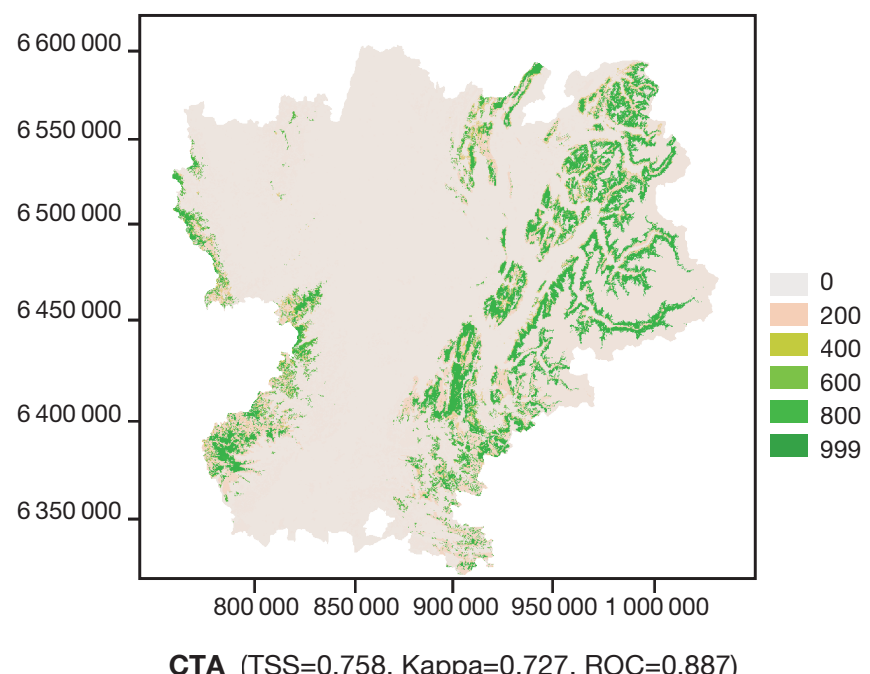

CTA (TSS $=0,758, \mathrm{Kappa}=0,727, \mathrm{ROC}=0,887$ )

FIG. 4. - Cartes de prédiction pour la Chevêchette d'Europe Glaucidium passerinum (Linnaeus, 1758) en Rhône-Alpes. Les six premiers modèles testés dans Biomod2 sont classés par ordre de qualité de prédiction décroissante (indices TSS, Kappa et ROC). Les modélisations utilisent 1229 points (pixels) de présences réelles et 1000 pseudo-absences générées selon la stratégie « disk » avec distance comprise entre 60 m et 20 km. Abréviations: ANN, artificial neural networks; CTA, classification tree analysis; FDA, flexible discriminant analysis; GBM, generalised boosted models; GLM, generalised linear models ; RF, random forests. Les valeurs de TSS (True Skill Statistic), de Kappa et de ROC (caractéristique du fonctionnement du récepteur en anglais) indiquent la performance des modèles: plus la valeur est proche de 1, meilleur est le modèle prédictif. En abscisse, la longitude et en ordonnée, la latitude (système de référence Lambert 93 en mètres). 
TABLEAU 4. - Contribution des différentes variables environnementales à la prédiction pour la Chevêchette d'Europe Glaucidium passerinum (Linnaeus, 1758) et la Chouette de Tengmalm Aegolius funereus (Linnaeus, 1758) selon le modèle Random Forest issu de Biomod2.

\begin{tabular}{|c|c|c|c|c|}
\hline \multirow[b]{2}{*}{ Variables environnementales } & \multicolumn{2}{|c|}{ Chevêchette d'Europe } & \multicolumn{2}{|c|}{ Chouette de Tengmalm } \\
\hline & $\begin{array}{l}\text { Pourcentage } \\
\text { de contribution }\end{array}$ & Classes préférentielles & $\begin{array}{l}\text { Pourcentage } \\
\text { de contribution }\end{array}$ & Classes préférentielles \\
\hline Altitude & $38 \%$ & 750 à $800 \mathrm{~m}$ & $35 \%$ & 900 à 1000 m \\
\hline Types d'occupation des sols & $34 \%$ & $\begin{array}{l}\text { Forêts fermées sapin ou épicéa } \\
\text { Forêts fermées conifères } \\
\text { Forêts fermées mélange } \\
\quad \text { feuillus-conifères } \\
\text { Forêts ouvertes }\end{array}$ & $32 \%$ & $\begin{array}{l}\text { Forêts fermées mélange } \\
\quad \text { feuillus-conifères } \\
\text { Forêts fermées sapin ou épicéa } \\
\text { Forêts fermées conifères } \\
\text { Forêts fermées feuillues }\end{array}$ \\
\hline Distance par rapport à une route & $10 \%$ & & $11 \%$ & \\
\hline $\begin{array}{l}\text { Distance par rapport à une zone } \\
\text { urbanisée }\end{array}$ & $8 \%$ & & $11 \%$ & \\
\hline Distance par rapport à une prairie & $8 \%$ & & $8 \%$ & \\
\hline Topographie & $2 \%$ & & $3 \%$ & \\
\hline
\end{tabular}

\section{Comparaison aux données de l'Atlas}

Les cartes issues de notre modélisation se superposent globalement bien à celles de l'Atlas des Oiseaux nicheurs. En particulier, la base de données que nous avons analysée ne contenait pas d'observation pour l'Est du Massif central mais la modélisation plaidait en faveur de secteurs favorables dans cette zone: les données de l'Atlas attestent que c'est bien le cas. À l'inverse, notre modélisation des secteurs potentiellement favorables n'englobe pas certains secteurs de présence avérée dans l'Atlas, comme par exemple les plateaux et collines du Bas-Dauphiné pour la Chevêchette d'Europe et les bois proches du Lac Léman pour la Chouette de Tengmalm. Dans ce cas, on pourrait conclure à une défaillance du modèle mais il s'agit dans les deux cas d'observations ponctuelles localisées en conditions marginales en terme d'altitude. En revanche, il est intéressant de noter que de nombreux secteurs sont prédits comme favorables sans donnée de présence, ni dans notre étude ni d'après l'Atlas. C'est par exemple le cas pour la Chevêchette d'Europe et la Chouette de Tengmalm dans le nord-est du Bas-Bugey, le massif du Grand Colombier, et pour la Chevêchette d'Europe dans le nord-est de la Drôme - secteur de Lus la Croix Haute et le sud-ouest de l'Ardèche. Enfin, pour la Chouette de Tengmalm, cela concerne aussi la vallée de Valoirette entre Valloire et le Galibier en Savoie, le nord des Baronnies dans la Drôme et le mont du Chat en Savoie. Il convient de vérifier auprès des experts réalisant des prospections si elles ont déjà fait l'objet de recherches infructueuses et mettent alors en défaut la robustesse de la prédiction, ou si elles mériteraient d'être prospectées.

\section{VÉRIFICATION DES CARTES DE PRÉDICTION} PAR LES GESTIONNAIRES D'ESPACES NATURELS

Dès fin 2016, des membres du Réseau PCM LPO-ONF implantés en Rhône-Alpes, ainsi que d'autres gestionnaires d'espaces naturels, ont disposé des cartes de probabilité de présence pour les confronter à leurs connaissances de terrain et tester leur utilité pour la recherche de nouveaux sites de présence.

La modélisation des habitats potentiels pour ces deux espèces a permis d'étendre les prospections en ciblant des secteurs où leur présence était présumée voire connue, mais de façon marginale. Depuis la réalisation de la modélisation pour la Chouette de Tengmalm, seule une année a été globalement favorable à sa reproduction et donc à sa détection, ce qui n'a pas encore permis d'apporter de nouvelles connaissances sur l'espèce.

\section{Réseau avifaune de l'Office national des Forêts}

La diffusion des cartes de prédiction issues de la modélisation, concomitamment aux deuxièmes rencontres nationales du groupe Petites Chouettes de Montagne à Allevard-les-Bains en Isère en 2016, a donné à l'Office national des Forêts l'opportunité de lancer une nouvelle campagne de prospections de terrain, ciblées dans la partie ouest du département (massifs de l'Épine-mont du Chat, de Chartreuse, de Belledonne et d'une partie des Bauges).

La recherche de la Chevêchette d'Europe a commencé en début d'hiver 2016, en se concentrant sur les zones de présence non encore recensées mais identifiées d'après la modélisation comme étant des habitats favorables avec une forte probabilité (>80 \%). Dynamisés par Sylvain Ducruet, correspondant de massif ONF Petites Chouettes de Montagne en Savoie, plusieurs forestiers de l'Unité territoriale de Chambéry, appuyés par deux stagiaires, Alex Vircondelet et Romain Ghiazza, formés au préalable aux techniques de prospections des petites chouettes de montagne par le réseau avifaune, ont parcouru des secteurs de forêt publique aux horaires favorables et dans les meilleures conditions météorologiques possibles, dans l'espoir de contacter les espèces. Le protocole consistait à réaliser des points d'inventaires espacés de 500 mètres, d'une durée de 5 min 30 s maximum, composés de phases d'écoute et de phases de repasse. Faute de détection de chant spontané durant la première minute, 30 secondes de repasse à l'ocarina étaient diffusées, et ce jusqu'à entendre une réponse et dans la limite de cinq essais au maximum.

Dans l'hiver 2016-2017, sept mâles chanteurs de Chevêchette d'Europe ont été contactés dans des zones où la présence de l'espèce n'était pas connue, à la fois sur le massif de l'Épine $(n=2)$, sur les contreforts sud du massif des Bauges $(n=1)$, mais également en Chartreuse $(n=1)$ et en Belledonne $(n=3)$ 
Chouette de Tengmalm

A

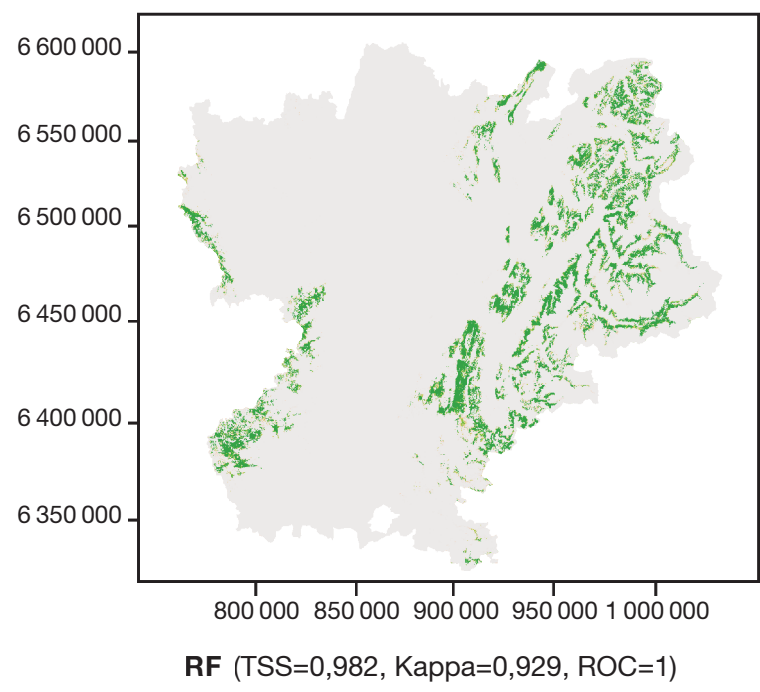

\section{B}

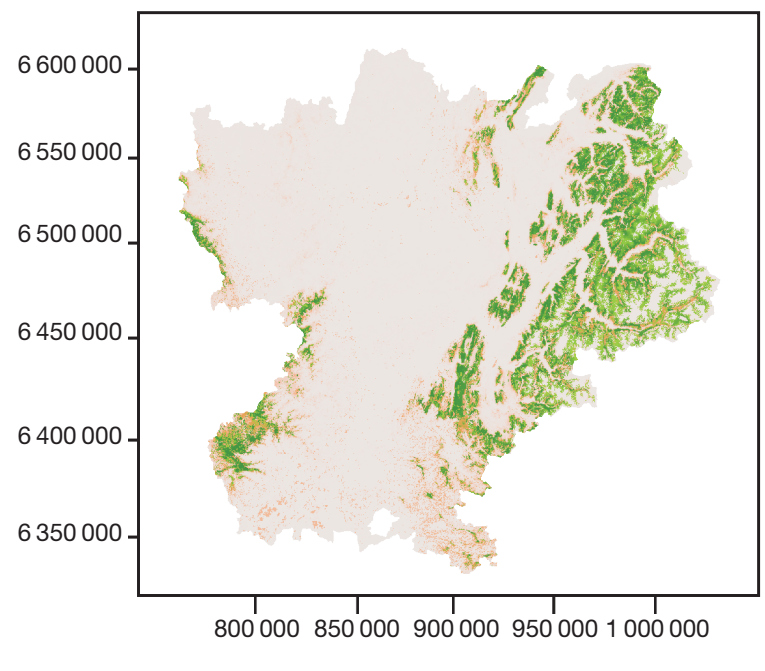

GBM (TSS=0,809, Kappa=0,704, ROC=0,954)

\section{Chevêchette d'Europe}
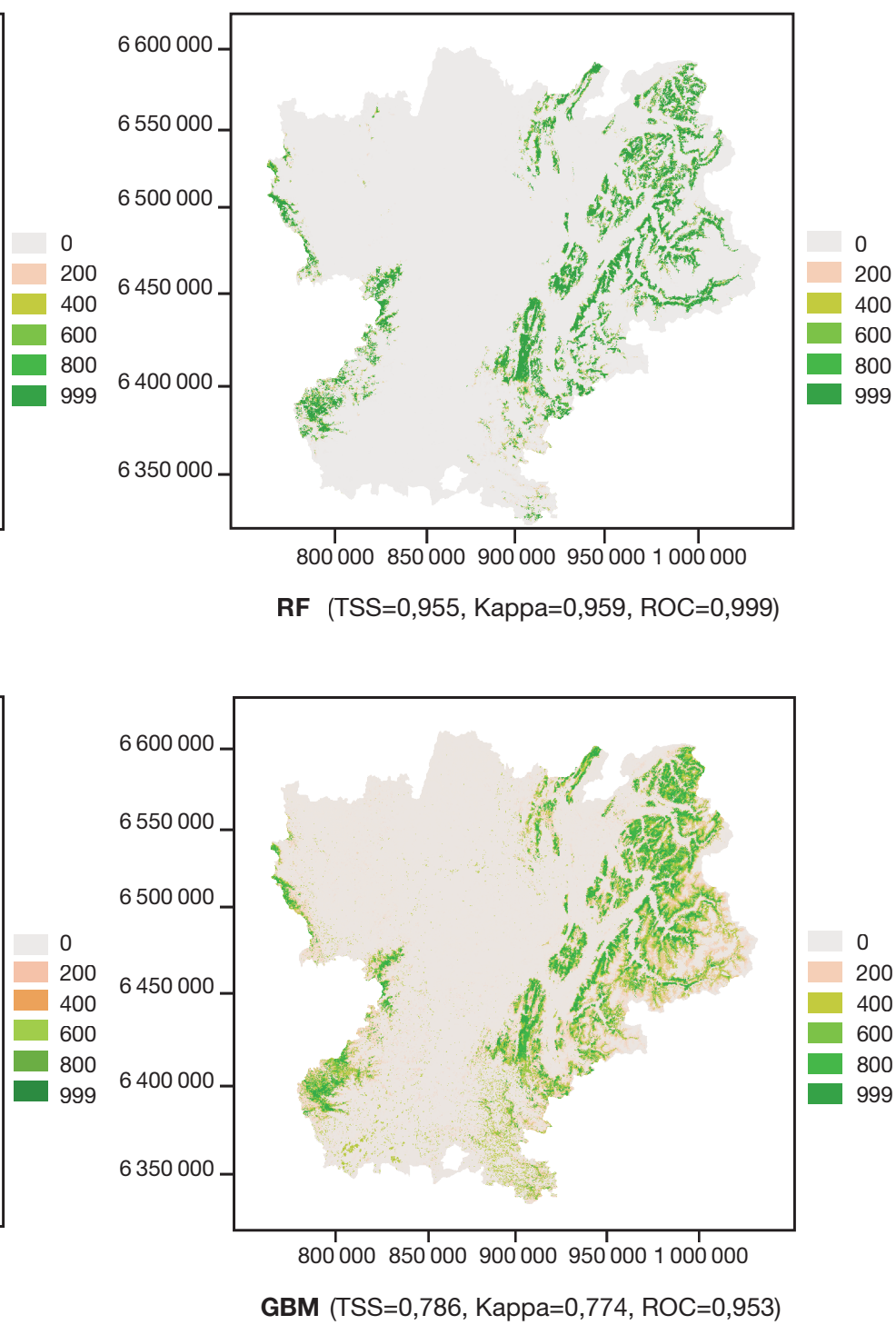

FIG. 5. - Cartes de prédiction de la répartition de la chouette de Tengmalm Aegolius funereus (Linnaeus, 1758) et de la Chevêchette d'Europe Glaucidium passerinum (Linnaeus, 1758) en Rhône-Alpes. Les deux meilleurs modèles issus de Biomod2 sont présentés. Le seuil de probabilité d'occurrence utilisé est de 80 \%. Les couleurs utilisées sont celles fournies par défaut pas Biomod. Abréviations: GBM, generalised boosted models; RF, random forests. Les valeurs de TSS (True Skill Statistic), de Kappa et de ROC (caractéristique du fonctionnement du récepteur en anglais) indiquent la performance des modèles: plus la valeur est proche de 1, meilleur est le modèle prédictif. En abscisse, la longitude et en ordonnée, la latitude (système de référence Lambert 93 en mètres).

savoyardes. Entre l'automne 2017 et le printemps 2018, huit nouvelles zones de présence étaient découvertes, dans le massif de l'Épine $(\mathrm{n}=5)$, sur les contreforts ouest et sud du massif des Bauges $(n=2)$, et dans la vallée des Huiles en Belledonne savoyarde $(\mathrm{n}=1)$.

Au vu des données de présence de Chevêchette d'Europe enregistrées dans la Base de Données naturalistes de l'ONF (BDN) en Savoie, il s'avère que seulement $10 \%$ des contacts (17 sur 168) ne sont pas situés dans la zone d'habitat favorable modélisée (Ghiazza 2018). Concaténées aux 222 données de présence en Tarentaise, on observe que $17 \%$ de ces contacts ont été obtenus entre 790 et 1100 m d'altitude, c'est-à-dire en deçà de la borne altitudinale inférieure suggérée par notre modélisation (Ghiazza 2018). Dans la partie centrale de la vallée de la Tarentaise, aucune observation n'a été réalisée en dehors de la zone modélisée (Mouchené D. comm. pers).

L'existence d'observations à plus basse altitude ou dans des zones non prédites comme favorables d'après la modélisation basée sur les observations réalisées entre 2005 et 2015 essentiellement hivernales peut s'expliquer par la probable extension occidentale de l'aire de présence de l'espèce, mais aussi avec la période de détection. En effet, les localisations d'automne réalisées au moment de la dispersion juvénile, peuvent correspondre à des habitats peu favorables de l'étage montagnard que parcourent de jeunes mâles, avant de s'installer dans une zone favorable pour passer l'hiver et se reproduire au printemps. 


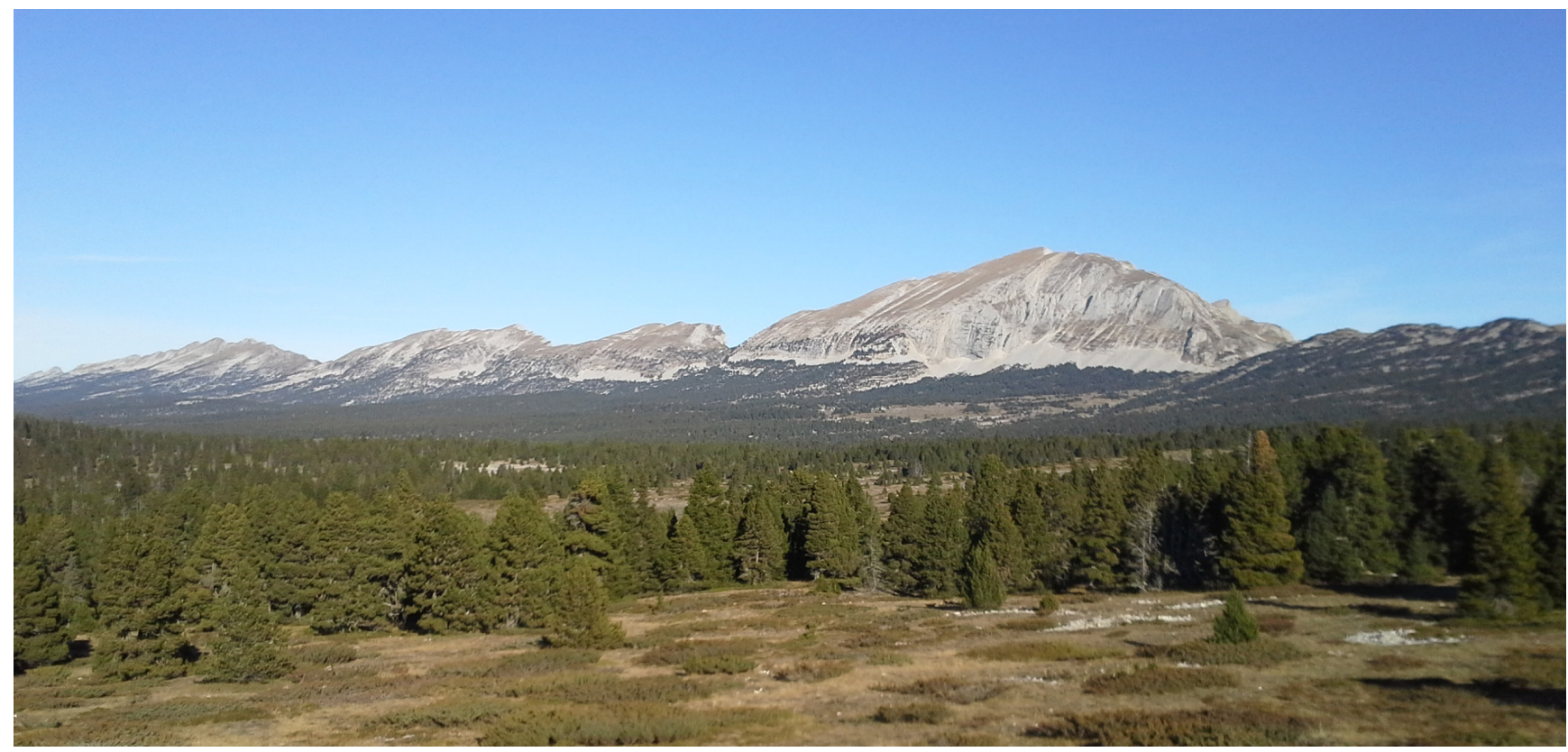

FIG. 6. - Habitat typique des petites chouettes de montagne sur les Hauts-Plateaux du Vercors. Crédit photo: H. Tournier.

\section{Parc naturel régional du Vercors}

Le parc du Vercors, gestionnaire de la Réserve naturelle nationale des Hauts-Plateaux du Vercors, a engagé il y a plus de dix ans un programme d'actions ciblées sur la conservation de la Chevêchette d'Europe. En effet, la présence d'une mosaïque riche de milieux forestiers et clairières intra-forestières, présentant des stades de maturation favorables aux petites chouettes de montagne, donnait à penser que la partie nord des Hauts-Plateaux était propice à accueillir une population de cette espèce (Fig. 6). Ainsi, la Réserve naturelle des Hauts-Plateaux et l'ONF ont mené une étude sur la Réserve biologique intégrale (RBI) du Vercors, en partenariat avec la LPO, entre 2009 et 2011, ayant pour objectif l'amélioration des connaissances sur l'écologie de la Chevêchette d'Europe. Le régime alimentaire, l'utilisation du domaine vital, le succès reproducteur, l'habitat de cette espèce sur le Vercors ont pu être précisément décrits (Barbaro et al. 2016).

À la suite de cette étude, le gestionnaire de la Réserve nationale a souhaité poursuivre le suivi de la Chevêchette d'Europe sur son territoire. L'objectif étant de mieux connaître sa distribution, deux transects ont été prospectés pendant cinq années sur la partie nord des Hauts-Plateaux, en appliquant un protocole de repasse puis d'écoute tous les $500 \mathrm{~m}$. Même si ces deux transects ont été établis a priori dans des habitats favorables à la Chevêchette d'Europe (car globalement identiques à la RBI), la carte de probabilité de présence issue de la modélisation nous a confortés dans le choix de ces emplacements. Il s'est d'ailleurs avéré que la Chevêchette d'Europe est bien présente dans la zone concernée, mais également dans le nord du massif.

À la suite de la première année de suivi de la partie nord, deux nouveaux transects ont été prospectés dans la partie sud des Hauts-Plateaux, l'un se trouvant en secteur favorable et l'autre non d'après notre modélisation. Les prospections n'ont révélé la présence d'aucune des deux espèces sur les deux secteurs, ne permettant pas de juger de la pertinence de la modélisation.

La parc envisage de déployer à l'échelle du massif du Vercors le protocole national de suivi "Tengmalm et Chevêchette", visant à évaluer la tendance d'évolution de leurs populations à long terme sur l'ensemble du territoire français. Ainsi, et grâce au partenariat établi avec la LPO, l'ONF et divers bénévoles, il est prévu de suivre une vingtaine de transects dispersés sur tout le massif. La modélisation des habitats favorables aux deux espèces a ainsi été utilisée en tant qu'outil d'aide à la décision quant au choix de l'emplacement de ces transects.

\section{LPO Auvergne-Rhône-Alpes}

Les cartes de modélisation du potentiel de présence des habitats (Fig. 5) ont dans un premier temps été mises sous format visuel et utilisable par les observateurs afin qu'ils puissent tous analyser les territoires favorables. Ces cartes, ainsi que les fichiers ayant permis de les générer, ont ensuite été diffusés auprès de chacune des antennes LPO de la région et des réseaux d'observateurs. L'objectif étant que chaque groupe d'observateurs repère les sites favorables sur lesquels orienter des prospections pour combler des absences de connaissances de l'espèce. Globalement dans les massifs alpins, si l'on n'a pas une connaissance exhaustive de tous les sites fréquentés, la présence de ces espèces est connue sur un nombre de sites maillant plutôt régulièrement le territoire (LPO Coordination Rhône-Alpes 2014).

Concernant le massif du Bugey, au sud du Jura, les cartes de modélisations ont servi de base pour définir 22 zones d'inventaire comprenant entre sept et 38 points d'écoute chacune. Sur 215 points d'écoute, la Chevêchette d'Europe a été contactée 
sur 106 points. Si les deux espèces y étaient connues avant les modélisations, les cartographies ont permis d'orienter les prospections. Elles ont permis de confirmer l'omniprésence de la Chevêchette d'Europe sur ce territoire et de réaffirmer le contexte favorable du massif du Bugey aux deux petites chouettes de montagne.

Plusieurs sites prédits comme potentiellement favorables sont à noter en dehors des Alpes, par exemple le chaînon du Salève (Haute-Savoie) sur lequel les deux espèces n'étaient pas connues et pour lequel les habitats apparaissent comme favorables à la présence de ces deux espèces. Ce chaînon, situé à mi-chemin entre les massifs du Jura et des Alpes, peut servir de relais pour des échanges de population. Il a été prospecté par les ornithologues locaux qui ont trouvé des individus chanteurs de Chevêchette d'Europe, mais seulement en période automnale. Aucune des prospections printanières réalisées jusqu'à maintenant n'a été concluante.

Dans la zone du Massif central, des prospections réalisées en parallèle de notre travail de modélisation ont révélé que la Chevêchette d'Europe n'y était pas rare. Il serait intéressant de faire un point sur la situation actuelle et de valider les modèles utilisés suite à l'ensemble de ces découvertes.

\section{DISCUSSION}

\section{APPORTS ET LIMITES DE L'APPROCHE PAR MODÉLISATION}

Avec l'accroissement du nombre de données naturalistes - professionnelles ou participatives - l'utilisation de la modélisation pour prédire la répartition potentielle des espèces connait un essor non négligeable depuis quelques années. Il est ainsi possible d'obtenir, sur la base de données existantes, des cartographies prédictives de la distribution d'espèces, y compris celles dont l'observation est difficile. En utilisant des données recueillies en Rhône-Alpes par des naturalistes et gestionnaires d'espaces naturels entre 2007 et 2014, nous avons modélisé la répartition potentielle des petites chouettes de montagne (Chevêchette d'Europe et Chouette de Tengmalm) sur l'ensemble de la région avec des résultats très satisfaisants au regard des métriques de diagnostic utilisées (TSS, Kappa, ROC) et de la correspondance avec les données - indépendantes - de l'Atlas national des Oiseaux (Muller \& Issa 2015). Ainsi, même avec des données parcellaires, assez mal réparties sur la zone d'étude et partiellement "opportunistes» sans plan d'échantillonnage prédéfini, il est possible de se faire une idée relativement précise de l'aire de répartition de ces espèces à l'échelle régionale, à l'instar d'autres cas d'étude à large échelle (Kelling et al. 2019).

Les différents tests ont montré que les modèles basés sur des données d'occurrence (i.e., présences et absences relevées) étaient moins performants que ceux basés sur les présences avérées complétées par des pseudo-absences. Ce résultat peut paraître assez surprenant a priori, mais il est possible que les données d'absence relevées par les observateurs de terrain soient en majorité des non-détections alors que l'espèce est effectivement présente (faux négatifs). Ces données d'absence erronées pourraient être dues à différents facteurs comme la grande discrétion de la Chouette de Tengmalm qui chante et répond à la repasse de façon parcimonieuse et aléatoire (Hedel \& Guérin 2019) et des conditions peu favorables à la reproduction (maigres ressources alimentaires ou conditions météorologiques peu favorables). Il est également très vraisemblable que toutes les données d'absence ne soient pas reportées, en particulier dans les secteurs considérés comme les moins propices. Au regard de ces incertitudes, les pseudoabsences, tirées au sort au hasard dans un rayon maximal de $20 \mathrm{~km}$ sont plus performantes. De même, le travail sur plusieurs années permet également de lisser les effets phénologiques et les difficultés de détection. La comparaison qualitative avec les données d'Atlas semble confirmer ces résultats, par exemple au sud de la région, alors qu'aucune donnée n'était disponible dans la base utilisée pour la modélisation (Figs 2; 5). Néanmoins, un secteur de présence en basse altitude de la Chouette de Tengmalm ne ressortait pas comme favorable à l'issue de la modélisation, faute d'avoir pu intégrer ces observations atypiques dans les analyses. Cela indique l'importance de bien couvrir l'ensemble des gradients écologiques et de la zone d'étude avant de procéder à la modélisation.

\section{INFLUENCE DES VARIABLES ENVIRONNEMENTALES}

Les modèles de distribution confirment l'influence prépondérante de l'altitude - et par extension du climat, notamment de la température - sur la répartition des petites chouettes de montagne (Castro et al. 2008; Redon 2012; Brambilla et al. 2015). En effet, ces deux oiseaux adaptés au froid sont considérés comme des espèces boréo-alpines dont les Alpes constituaient jusqu'à récemment la limite sud de l'aire de répartition, hormis, en France, les Pyrénées (Sordello 2012). Les conditions météorologiques rencontrées en moyenne et en haute montagne (jusqu'à $1850 \mathrm{~m}$ pour la Chouette de Tengmalm et $2200 \mathrm{~m}$ pour la Chevêchette d'Europe dans notre cas) sous les latitudes métropolitaines correspondent aux conditions rencontrées plus au cœur de l'aire de répartition de ces deux espèces (Korpimaki 1993).

De plus, nos résultats montrent que l'occupation du sol constitue la seconde variable la plus influente de la prédiction. Pour mémoire, environ $90 \%$ des observations des deux espèces étaient localisées sur des sites correspondant à des forêts fermées de conifères, de feuillus et mixtes alors qu'elles ne représentent respectivement que $16 \%, 20 \%$ et $4 \%$ de la zone d'étude. Les deux chouettes sont des espèces forestières qui nichent dans des cavités creusées par des pics (Cockle et al. 2011 ; Zellweger et al. 2013; Barbaro et al. 2016). Elles bénéficient ainsi de mosaïques de peuplements plutôt matures et riches en gros et vieux arbres, voire peut-être de peuplements plus jeunes mais dépérissants, qui présentent dans les deux cas de nombreuses cavités, si possible proches, aptes à accueillir des nichées et à servir de garde-manger (Korpimaki 1993; Mariné \& Dalmau 2000). La Chevêchette d'Europe chasse préférentiellement en lisière de forêt où elle se nourrit de rongeurs et de passereaux (Strøm \& Sonerud 2001; Barbaro et al. 2016). Elle semble également réagir favorablement à la structuration verticale de la végétation, notamment les variations de hauteur et les lisières internes (Zellweger et al. 2013). Cependant, ce résultat doit être nuancé car, dans notre 
approche, la distance aux pâtures ne contribue que pour environ $8 \%$ à l'ajustement du meilleur modèle, ce qui peut s'expliquer par le fait que ces oiseaux se nourrissent plutôt en bordure de forêt qu'au centre des milieux ouverts comme d'autres rapaces. Il est possible que le «variable distance à la plus proche prairie» ne rende qu'imparfaitement compte de la disponibilité en espaces ouverts à proximité. Les analyses pourraient être complétées avec d'autres métriques paysagères décrivant l'habitat à l'échelle du territoire, comme la proportion de milieux ouverts dans un certain rayon ou encore des indices de connectivité des milieux ouverts. Néanmoins, malgré l'utilisation de variables relativement sommaires, les modèles sont globalement cohérents avec la biologie de ces deux espèces et l'aire de répartition potentielle s'ajuste globalement bien avec les données d'Atlas.

En résumé, si l'on cherche à définir les caractéristiques de ces zones à partir des deux variables ayant le plus de poids, on peut déduire de ces cartes de prédiction que les habitats propices à la Chevêchette d'Europe sont majoritairement situés à plus de $800 \mathrm{~m}$ d'altitude et sont associés à des peuplements fermés à base de coniferes, ou mixtes, mais pas de feuillus dominants. Pour la Chouette de Tengmalm, les habitats favorables se rencontrent au-dessus de 900 à 1000 m d'altitude dans des peuplements fermés qu'ils soient composés de feuillus, de conifères ou mixtes. Cependant il serait intéressant, pour compléter l'approche, de disposer de données plus précises de structure forestière (Zellweger et al. 2013; Barbaro et al. 2016) afin d'évaluer la part respective des facteurs topographiques et climatiques et de la structure forestière. Il serait ainsi possible d'améliorer encore leur prise en compte dans la gestion forestière.

\section{PeRsPeCTIVES}

Malgré des résultats encourageants, la modélisation de l'habitat et de la répartition des petites chouettes de montagne reste perfectible et encourage à l'amélioration de la qualité des données d'entrée relatives aux espèces et à la structure forestière et paysagère. Ces dernières années, la généralisation de l'utilisation du GPS et des outils de visualisation cartographique a permis d'améliorer grandement la géolocalisation des observations, même en contexte forestier. Dans le jeu de données ONFLPO, la précision de la localisation de nombreuses données antérieures à 2007 se limitait à la commune. Plusieurs autres perspectives d'amélioration se dessinent.

La première perspective qu'offre l'existence d'une cartographie à l'échelle de la région est une meilleure structuration du plan d'échantillonnage en termes de couverture spatiale régionale, incluant des situations extrêmes, notamment en limites altitudinales. La cartographie permet de stratifier les zones en fonction de la probabilité de présence des deux espèces et ainsi mieux couvrir les gradients des facteurs altitude et peuplements forestiers qui influencent leur répartition. Une telle stratification pourrait également améliorer la standardisation du protocole de suivi.

Comme mentionné plus haut, les deux espèces sont difficiles à contacter et il est fréquent de devoir passer plusieurs fois avant d'obtenir des contacts. La modélisation de cette détectabilité imparfaite pourrait améliorer l'estimation de la probabilité de présence, notamment s'il s'avère que cette détectabilité dépend de l'environnement (peut-être ces chouettes sont-elles plus difficiles à détecter dans des habitats sub-optimaux) ou de l'effectif (les individus sont généralement plus détectables les années de fortes densités, quand la compétition intra-spécifique est plus importante). Pour modéliser cette détectabilité, il faut répéter les passages au cours d'une même année, un protocole qui engendre un surcoût d'échantillonnage et auquel des bénévoles peuvent ne pas adhérer. Cette approche répétée d'une année sur l'autre permettrait de faire la part entre les variations inter-annuelles de détectabilité de les fluctuations des niveaux des populations connues pour être très importantes (Hakkarainen et al. 1997a; Henrioux et al. 2016).

Par ailleurs, il conviendrait de compléter spatialement notre jeu de données en couvrant mieux la zone d'étude (en particulier à l'ouest) et en intégrant des observations y compris dans des situations a priori très peu favorables. Dans cette optique, il serait certainement utile d'analyser conjointement des données obtenues selon un protocole standardisé et des données opportunistes.

Les variables environnementales explicatives de la répartition des petites chouettes de montagne pourraient également être précisées et enrichies. Il serait intéressant d'inclure des indices d'hétérogénéité paysagère permettant de mieux décrire la mosaïque locale et des prédicteurs biotiques comme la stratification verticale et horizontale des peuplements, l'abondance d'arbres morts et microhabitats favorables tels que les cavités (Zellweger et al. 2013). À l'instar des travaux lancés en collaboration avec le Parc national des Cévennes (Guérin \& Laguet 2019), la connaissance précise des sites de nidification et leur description pourraient également permettre de mieux comprendre la sélection des habitats par ces deux petites chouettes et les facteurs influençant leur présence de manière plus fine à l'intérieur des peuplements forestiers (Hakkarainen et al. 1997b; Henrioux et al. 2016).

Une autre piste qui pourrait permettre d'améliorer à la fois les prédictions et les connaissances sur la biologie des espèces serait la combinaison de données d'espèces avec des données numériques de type Lidar pour décrire l'environnement local (à la fois paysager et forestier) à plus ou moins large échelle autour des points de relevés, ou encore de l'interprétation de données satellites plus précises que CORINE (Ingalda et al. 2017). L'utilisation d'une combinaison de données de terrain et de données numériques a par ailleurs déjà fait ses preuves pour d'autres espèces rares et cryptiques comme pour deux espèces de chauve-souris (Le Roux et al. 2017) ou pour le Pic tridactyle, Picoides tridactylus (Linnaeus, 1758) (Zielewska-Büttner et al. 2018). Enfin, certaines publications mentionnent également que l'inclusion d'indices de présence d'autres espèces dont les chouettes dépendent, notamment des pics (Joveniaux \& Durand 1987; Ravussin et al. 1994; Brambilla et al. 2013), la réaction des passereaux à la repasse (Dutour et al. 2016), ou la présence de Chouette hulotte, Strix aluco (Linnaeus, 1758), potentielle prédatrice des deux chouettes de montagne, permet d'améliorer significativement les prédictions. Il s'agirait par exemple d'établir des cartes 
d'abondance des pics, comme nous l'avons fait pour les chouettes, puis d'utiliser ces données de distribution comme variables explicatives. Dans le cas où la repasse entraîne une réponse agressive de passereaux ou le chant de la chouette hulotte, il s'agirait de considérer ces réactions comme des données de présence, avec le même poids dans l'analyse que des présences avérées (chant ou observation directe) ou bien un poids inférieur.

L'ensemble de ces propositions questionnent le compromis entre quantité et précision des données et qualité finale de la modélisation. Il conviendrait de tester les différentes options évoquées et d'évaluer leur rapport coût/bénéfice au regard des attentes des gestionnaires et des naturalistes, de leur utilisation potentielle, ainsi que des exigences de suivi des espèces. Si la cartographie que nous proposons, basée sur des données environnementales relativement simples à acquérir, semble pertinente, il convient néanmoins d'envisager des améliorations méthodologiques de manière à assurer un suivi spatial et temporel pertinent et précis, c'est-à-dire capable de détecter des évolutions de populations à court terme. À ce titre, une extension de l'approche à d'autres zones biogéographiques comme les Pyrénées pourrait s'envisager (Potrony et al. 2019). Enfin, il serait intéressant d'intégrer une composante climatique dans la modélisation. Il s'agirait d'étudier dans quelle mesure le climat est un bon ou mauvais prédicteur du niveau des populations d'une année sur l'autre, mais aussi dans quelle mesure l'évolution prévisible des ceintures forestières en montagne sous l'effet du changement climatique risque de fragmenter la distribution de ces chouettes.

\section{Remerciements}

Nous remercions l'ensemble des contributeurs à la collecte des données sur les petites chouettes de montagne. Sans leur assiduité et leur dévotion aux inventaires naturalistes, de telles approches ne seraient pas possibles. Nos remerciements vont également à Fabienne Henrioux et Luc Barbaro, relecteurs de cet article, qui ont contribué de façon constructive à l'améliorer.

\section{RÉFÉRENCES}

Barbaro L., Blache S., Trochard G., Arlaud C., Lacoste (de) N. \& KAYSER Y. 2016. - Hierarchical habitat selection by Eurasian Pygmy Owls Glaucidium passerinum in old-growth forests of the southern French Prealps. Journal of Ornithology 157: 333-342. https://doi.org/10.1007/s10336-015-1285-3

BARTHOD C. 2015. - La multifonctionnalité des forêts entre discours et pratiques: illusion ou réalité à assumer? Revue forestière française 67 (4): 293-319. https://doi.org/10.4267/2042/59287

Brambilla M., Bergero V., Bassi E. \& Falco R. 2015. — Current and future effectiveness of Natura 2000 network in the central Alps for the conservation of mountain forest owl species in a warming climate. European Journal of Wildlife Research 61: 35-44. https://doi.org/10.1007/s10344-014-0864-6

Brambilla M., Bassi E., Bergero V., Casale F., Chemollo M., Falco R., Longoni V., Saporetti F., Viganò E. \& Vitulano S. 2013. - Modelling distribution and potential overlap between Boreal Owl Aegolius funereus and Black Woodpecker Dryocopus martius: implications for management and monitoring plans.
Bird Conservation International 23 (4): 502-511. https://doi. org/10.1017/S0959270913000117

Castro A., Muñoz A. R. \& Real R. 2008. - Modelling the spatial distribution of the Tengmalm's owl Aegolius funereus in its Southwestern Palaeartic limit (NE Spain). Ardeola 55: 71-85.

Cockle K. L., Martin K. \& Wesołowski T. 2011. — Woodpeckers, decay, and the future of cavity-nesting vertebrate communities worldwide. Frontiers in Ecology and the Environment 9 (7): 377-382. https://doi.org/10.1890/110013

DutOur M., LeNA J.-P. \& LeNGAGNE T. 2016. — Mobbing behaviour varies according to predator dangerousness and occurrence. Animal Behaviour 119: 119-124. https://doi.org/10.1016/j. anbehav.2016.06.024

GHiazZA R. 2018. - Prospection de la Chouette Chevêchette dans les forêts publiques de Savoie 2016/2018. Rapport de stage ONF. Office national des Forêts, Chambéry, $26 \mathrm{p}$.

Guérin B. \& Laguet S. 2019. - Caractérisation des habitats du Pic noir (Dryocopus martius) et de la Chouette de Tengmalm (Aegolius funereus) en forêt domaniale de l'Aigoual, in Parc national des Cévennes, ONF \& LPO (éds), 3 e rencontres nationales du groupe Petites chouettes de montagne, Florac, 17-19 octobre 2019. Réseau avifaune ONF, Florac: 3-4.

HAKKarainen H., KoIVUnen V. \& KorpimäKI E. 1997a. — Reproductive success and parental effort of Tengmalm's owls: effects of spatial and temporal variation in habitat quality. Ecoscience 4 (1):35-42. https://doi.org/10.1080/11956860.1997.11682374

Hakkarainen H., KorpimäKi E., KoIvunen V. \& Kurki S. 1997b. - Boreal owl responses to forest management: a review. Journal of Raptor Research 31 (2): 125-128.

HEDEL A. \& GUÉRIN B. 2019. - Apports d'un enregistreur autonome pour l'étude du chant de la chouette de Tengmalm, in Parc national des Cévennes, ONF \& LPO (éds), 3e rencontres nationales du groupe Petites chouettes de montagne, Florac, 17-19 octobre 2019. Réseau avifaune ONF, Florac: 13-15.

Henrioux P., Henrioux J. D., Walder P. \& Henrioux F. 2016. - The Pygmy Owl Glaucidium passerinum in the Swiss Jura Mountains. Alauda 84: 253-266.

Ingalda J., Vincent A., Arias M., Tardy B., Morin D. \& RODES I. 2017. - Operational high resolution land cover map production at the country scale using satellite image time series. Remote Sensing 9 (1): 95. https://doi.org/10.3390/rs9010095

JOVENIAUX A. \& DURAND G. 1987. - Gestion forestière et écologie des populations de Chouette de Tengmalm (Aegolius funereus) dans l'Est de la France. Revue d'Écologie (La Terre et la Vie) 42 (Suppl. 4): 83-96.

Kelling S., Johnston A., Bonn A., Fink D., Ruiz-Gutierrez V., BONNEY R., Fernandez M., HOCHACHKA W. M., Julliard R., KraEmer R. \& GURAlNicK R. 2019. - Using semistructured surveys to improve citizen science data for monitoring biodiversity. Bioscience 69 (3): 170-179. https://doi.org/10.1093/biosci/biz010

KORPIMAKI E. 1993. - Does nest-hole quality, poor breeding success or food depletion drive the breeding dispersal of Tengmalm's owls? Journal of Animal Ecology 62: 606-613. https:// doi.org/10.2307/5382

LAGUET S. \& BeSNARD A. 2019. - Protocole national de suivi des tendances des effectifs des populations de petites chouettes de montagne sur sites de références - point d'avancement 2019, in Parc national des Cévennes, ONF \& LPO (éds), 3 e rencontres nationales du groupe Petites chouettes de montagne, Florac, 17-19 octobre 2019. Réseau avifaune ONF, Florac: 12-13.

Le Roux M., Redon M., Archaux F., Long J., Vincent S. \& LUQUE S. 2017. - Conservation planning with spatially explicit models: a case for horseshoe bats in complex mountain landscapes. Landscape Ecology 32: 1005-1021. https://doi.org/10.1007/ s10980-017-0505-z

Ligue pour la Protection des Oiseaux 2019. - Les Cahiers de la Surveillance. Rapaces. Bilan 2019. LPO, Rochefort.

LPo COORDINATION RHÔNE-Alpes 2014. — État des connais- 
sances sur les petites chouettes de montagne en Rhône-Alpes. Le Bièvre 26: 38-53.

Mariné R. \& Dalmau J. 2000. - Habitat use by Tengmalm's Owls Aegolius funereus in Andorra (eastern Pyrenees) during the breeding period. Ardeola 47: 29-36.

Muller Y. \& IsSA N. 2015. - Atlas des oiseaux de France métropolitaine - Nidification et présence hivernale. Delachaux et Niestlé, Paris, 1408 p.

Potrony D., Badosa E. \& CAmprodon J. 2019. — Modélisation de la distribution de la Chouette de Tengmalm à l'échelle des Pyrénées, in Parc national des Cévennes, ONF \& LPO (éds), 3 rencontres nationales du groupe Petites chouettes de montagne, Florac, 17-19 octobre 2019. Réseau avifaune ONF, Florac: 15.

R Core TeAm 2015. - R: A Language and Environment for Statistical Computing. R Foundation for Statistical Computing, Vienna, Austria.

Ravussin P.-A., Walder P., Henrioux P., Chabloz V. \& MenETREY Y. 1994. - Répartition de la Chouette de Tengmalm (Aegolius funereus) dans les sites naturels du Jura vaudois (Suisse). Nos Oiseaux 42: 245-260.

REDON M. 2012. - Biodiversité potentielle danse les forêts du Vercors: une approche hiérarchique pour la conservation des espaces forestiers. Irstea, Université de Grenoble, 195 p.

SORDELLO R. 2012. - Synthèse bibliographique sur les traits de vie de la Chouette de Tengmalm (Aegolius funereus (Linnaeus, 1758)) relatifs à ses déplacements et à ses besoins de continuités écologiques. Service du patrimoine naturel du Muséum national d'Histoire naturelle, Paris, $18 \mathrm{p}$.

STrøm H. \& Sonerud G. A. 2001. - Home range and habitat selection in the Pygmy Owl Glaucidium passerinum. Ornis Fennica 78: 145-158.

Thuillier W., Lafourcade B. \& Araujo M. 2010. - Presentation Manual for BIOMOD. [Manuel technique] LECA, UJF, Grenoble, 35 p.

Thuiller W., Lafourcade B., Engler R. \& Araújo M. B. 2009. - BIOMOD - a platform for ensemble forecasting of species distributions. Ecography 32 (3): 369-373. https://doi. org/10.1111/j.1600-0587.2008.05742.x

UICN FRANCE, MNHN, LPO, SEOF \& ONCFS 2016. La Liste rouge des espèces menacées en France - Chapitre Oiseaux de France métropolitaine. UICN France, Paris, $32 \mathrm{p}$.

ZeLlWEgER F., BRAUNisCH V., BALTENSWEILER A. \& BOLLMANN K. 2013. - Remotely sensed forest structural complexity predicts multi species occurrence at the landscape scale. Forest Ecology and Management 307: 303-312. https://doi.org/10.1016/j.foreco.2013.07.023

Zielewska-BÜtTNer K., Heurich M., MÜller J. \& Braunisch V. 2018. - Remotely sensed single tree data enable the determination of habitat thresholds for the three-toed woodpecker (Picoides tridactylus). Remote Sensing 10 (12): 1972. https://doi. org/10.3390/rs10121972 